\title{
STAR FORMATION AND FEEDBACK: A MOLECULAR OUTFLOW-PRESTELLAR CORE INTERACTION IN L1689N
}

\author{
D. C. Lis ${ }^{1,2}$, H. A. Wootten ${ }^{3}$, M. Gerin ${ }^{4}$, L. Pagani ${ }^{1}$, E. RouefF ${ }^{5}$, F. F. S van Der TaK ${ }^{6,7}$, C. Vastel ${ }^{8}$, and C. M. Walmsley ${ }^{9,10}$ \\ ${ }^{1}$ LERMA, Observatoire de Paris, PSL Research University, CNRS, Sorbonne Universités, UPMC Univ. Paris 06, F-75014 Paris, France; darek.lis@ obspm.fr \\ ${ }^{2}$ California Institute of Technology, Cahill Center for Astronomy and Astrophysics 301-17, Pasadena, CA 91125, USA \\ ${ }^{3}$ National Radio Astronomy Observatory, Charlottesville, VA 22903, USA; awootten@nrao.edu \\ ${ }^{4}$ LERMA, Observatoire de Paris, PSL Research University, CNRS, Sorbonne Universités, UPMC Univ. Paris 06, \\ École normale supérieure, F-75005, Paris, France; maryvonne.gerin@1ra.ens.fr \\ ${ }^{5}$ LERMA, Observatoire de Paris, PSL Research University, CNRS, Sorbonne Universités, UPMC Univ. Paris 06, F-92190 Meudon, France \\ ${ }^{6}$ SRON Netherlands Institute for Space Research, Landleven 12, 9747 AD Groningen, The Netherlands \\ ${ }^{7}$ Kapteyn Astronomical Institute, University of Groningen, The Netherlands \\ ${ }^{8}$ Institut de Recherche en Astrophysique et Planetologie, F-31028 Toulouse Cedex 4, France \\ ${ }^{9}$ INAF-Osservatorio astrofisico di Arcetri, Largo E. Fermi 5, I-50125 Firenze, Italy \\ ${ }^{10}$ Dublin Institute of Advanced Studies, Fitzwilliam Place 31, Dublin 2, Ireland \\ Received 2016 March 14; revised 2016 May 2; accepted 2016 May 3; published 2016 August 16
}

\begin{abstract}
We present Herschel, ${ }^{11}$ ALMA Compact Array (ACA), and Caltech Submillimeter Observatory observations of the prestellar core in L1689N, which has been suggested to be interacting with a molecular outflow driven by the nearby solar-type protostar IRAS 16293-2422. This source is characterized by some of the highest deuteration levels observed in the interstellar medium. The change in the $\mathrm{NH}_{2} \mathrm{D}$ line velocity and width across the core provides clear evidence of an interaction with the outflow, traced by the high-velocity water emission. Quiescent, cold gas characterized by narrow line widths is seen in the NE part of the core, while broader, more disturbed line profiles are seen in the $\mathrm{W} / \mathrm{SW}$ part. Strong $\mathrm{N}_{2} \mathrm{D}^{+}$and $\mathrm{ND}_{3}$ emission is detected with ACA extending $\mathrm{S} / \mathrm{SW}$ from the peak of the single-dish $\mathrm{NH}_{2} \mathrm{D}$ emission. The ACA data also reveal the presence a compact dust continuum source with a mean size of $\sim 1100$ au, a central density of $(1-2) \times 10^{7} \mathrm{~cm}^{-3}$, and a mass of $0.2-0.4 M_{\odot}$. The dust emission peak is displaced $\sim 5^{\prime \prime}$ to the south with respect to the $\mathrm{N}_{2} \mathrm{D}^{+}$and $\mathrm{ND}_{3}$ emission, as well as the single-dish dust continuum peak, suggesting that the northern, quiescent part of the core is characterized by spatially extended continuum emission, which is resolved out by the interferometer. We see no clear evidence of fragmentation in this quiescent part of the core, which could lead to a second generation of star formation, although a weak dust continuum source is detected in this region in the ACA data.
\end{abstract}

Key words: ISM: molecules - stars: formation - submillimeter: ISM - techniques: interferometric - techniques: spectroscopic

\section{INTRODUCTION}

Low-mass star formation is known to occur exclusively in the shielded interiors of molecular cloud cores, when gravity wrests control from supporting thermal, magnetic, and turbulent pressures and collapse ensues. Over the past 20 years, much effort has been focused on understanding the subsequent stages of star formation, when a central object and a surrounding circumstellar disk are formed and evolve toward a newly formed star and, potentially, its associated planetary system. However, the initiation of this process is among the least understood steps of star formation. Yet it is key to understanding some of its most fundamental aspects, such as the initial mass function, the binarity fraction and its dependence on stellar mass, and the star formation efficiency.

Generally, starless cores are thought to represent this earliest stage of star formation. In one view, magnetically supported clouds develop magnetically critical cores over a long timescale (a few Myr) in a quasi-static fashion through the process of ambipolar diffusion (Shu et al. 1987; Mouschovias et al. 1991). Alternatively, turbulence continuously shreds and twists molecular clouds, forming denser structures-through shocks - that collapse, fragment, or otherwise disappear. In this view,

\footnotetext{
11 Herschel is an ESA space observatory with science instruments provided by European-led Principal Investigator consortia and with important participation from NASA.
}

starless cores are highly dynamical structures that evolve over a few sound-crossing times, i.e., 10 times faster than the ambipolar diffusion timescale (see Ballesteros-Paredes \& Hartmann 2007 and references therein). These different scenarios for the formation and evolution of starless cores result in very different three-dimensional (3D) morphological structures and unique dynamic signatures. The environment in which a core forms is also critical to its subsequent evolution. Dense cores in regions of cluster-forming clouds tend to have higher masses and column densities than isolated prestellar cores. The molecular cloud cores in Taurus and Ophiuchus provide a case in point (e.g., Ward-Thompson et al. 2007 and references therein).

Much of our insight into the structure of starless cloud cores comes from observations of millimeter dust emission (Bergin \& Tafalla 2007). Herschel observations using PACS and SPIRE have shown that cores preferentially form along dense filaments (André et al. 2010; Molinari et al. 2010). The Herschel Gould belt survey in particular has discovered a large population of nearby prestellar cores, allowing for the accurate determination of the core mass function (e.g., Könyves et al. 2010; André et al. 2014, but see the recent results of Pagani et al. 2015), which can be compared with model predictions for the IMF produced by the collapse of a turbulent cloud (e.g., Hennebelle \& Chabrier 2008, 2009). High angular resolution interferometric continuum studies provide insights 
into the formation of multiple systems (Maury et al. 2010). However, continuum observations are likely to yield only a partial picture of the cloud structure since coagulation of dust is a key process at the high densities of inner starless cores, and this will change the grain opacity coefficient and effectively "hide" much of the mass of the dust from view (Pagani et al. 2010; Steinacker et al. 2010; Lefèvre et al. 2014, 2016). Moreover, dust studies do not provide direct insight into the dynamics of these cores or their chemistry.

Likewise, molecular observations are also generally known to provide a biased view of starless cores. This reflects the condensation of species onto ice mantles at high densities. There are two exceptions to this general rule. First, for reasons that are not fully understood, nitrogen-bearing species, ammonia in particular, do not seem to participate in this freeze out (Tafalla et al. 2002; Crapsi et al. 2007). Deuterated species form the second exception (see the recent review by Ceccarelli et al. 2014). The high deuteration of gas-phase species is, in effect, a result of the freeze out and the coincidental disappearance of ortho- $\mathrm{H}_{2}$ from the gas phase, without which no deuteration would happen (Pagani et al. 1992, 2009; Flower et al. 2006). This drives up the gas-phase abundance and fractionation of $\mathrm{H}_{3}^{+}$. The high fractionation of this species is then passed on through ion-molecule chemistry, to the few molecules remaining in the gas phase (Roberts \& Millar 2000). These chemical aspects can be used to our advantage to study prestellar cores. We note that some deuterated species, such as formaldehyde or water, are formed and deuterated primarily through grain-surface chemistry in icy mantles (Ceccarelli et al. 2014). These molecules can then be thermally or photodesorbed in active environments, such as molecular hot cores, outflows, or shocks. Of the two key deuterated species studied here, $\mathrm{N}_{2} \mathrm{D}^{+}$is believed to be formed in cold, dense, $\mathrm{CO}$ depleted regions through gas-phase chemistry driven by $\mathrm{H}_{3}^{+}$ and its deuterated isotopologues. Ammonia deuteration is also straightforward to understand in the framework of gas-phase processes (Roueff et al. 2005). However, recent laboratory experiments (Fedoseev et al. 2015) suggest that ammonia and its deuterated isotopologues may also be formed thorough successive additions of hydrogen and deuterium atoms to nitrogen atoms in CO-rich interstellar ice analogs, which are efficient at temperatures below $15 \mathrm{~K}$.

High spectral resolution investigations of the velocity field in central regions of cold, dense cores are invaluable for disentangling rotation and collapse (Belloche et al. 2002), providing excellent constraints on fragmentation and formation of disks, which are critically dependent on the magnetic field (Hennebelle \& Fromang 2007; Hennebelle \& Teyssier 2007). This requires, however, that suitable molecular tracers be chosen. The fundamental submillimeter lines of ammonia isotopologues have critical densities of the order of $10^{6} \mathrm{~cm}^{-3}$ and, with simple hyperfine patterns, are excellent tracers of dense gas at the onset of star formation (Lis et al. 2002b, 2006), providing information complementary to $\mathrm{H}_{2} \mathrm{D}^{+}$(van der Tak et al. 2005; Vastel et al. 2006, 2012; Caselli et al. 2008). The interferometric study of Crapsi et al. (2007) has shown a very high $\mathrm{NH}_{2} \mathrm{D} / \mathrm{NH}_{3}$ fractionation ratio in the central region of L1544, $0.5 \pm 0.2$ which demonstrates the persistence of deuterated ammonia molecules at very high densities $\left(\sim 2 \times 10^{6} \mathrm{~cm}^{-3}\right)$, and low temperatures $(\sim 10 \mathrm{~K})$. Very high deuteration ratios have also been reported in other molecules (e.g., $\mathrm{N}_{2} \mathrm{D}^{+} / \mathrm{N}_{2} \mathrm{H}^{+}=0.7 \pm 0.2$ in L183; Pagani et al. 2007; see the recent review of Ceccarelli et al. 2014). Nevertheless, lines of deuterated isotopologues of ammonia, with their simple hyperfine patterns, fulfill all of the requirements for being optimum tracers of cold, dense cores.

Herschel has enabled for the first time systematic studies of the fundamental submillimeter lines of $\mathrm{NH}_{3}$ and $\mathrm{NH}_{2} \mathrm{D}$ in prestellar cores. The long-term goals of this study are threefold: (a) to firmly establish the utility of ammonia isotopologues as tracers of dense, cold gas at the onset of star formation as postulated by Lis et al. (2006, 2008); (b) to determine the morphology and velocity structure of an example prestellar core, the processes that drive the evolution in this earliest stage of star formation and that control such important aspects as core fragmentation, and the resulting initial mass function and binarity fraction of protostars; (c) to improve our understanding of the chemical and physical processes that control gas-grain interaction, freeze out, and mantle ejection in dense cloud cores, including when/why species freeze out, and of deuterium fractionation. These processes are of key interest for the chemistry of regions of star formation, and hence the supply of volatiles in planet-forming environments, likely including the early solar nebula.

In this paper, we present extensive single-dish and interferometric observations of L1689N, a nearby (120 pc; Loinard et al. 2008) dark cloud located in the $\rho$ Ophiuchi complex which harbors one of the best-studied solar-type protostars, IRAS 16293-2422 (hereafter IRAS 16293). The nearby protostellar core, sometimes referred to as I16293E, has previously been suggested to be interacting with the blue lobe of one of the outflows driven by IRAS 16293 (Wootten \& Loren 1987; Lis et al. 2002a; Stark et al. 2004). This region is thus an excellent laboratory in which to study the effects of stellar feedback on the physics and chemistry of the surrounding interstellar medium (ISM). In addition to Herschel observations, we have obtained interferometric observations of the $970 \mu \mathrm{m}$ dust continuum, $\mathrm{ND}_{3}$ and $\mathrm{N}_{2} \mathrm{D}^{+}$line emission using the ALMA Compact Array (ACA), as well as maps of several deuterated molecular tracers using the Caltech Submillimeter Observatory (CSO). The observational results are presented in Section 3, followed by a discussion in Section 4, and a summary in Section 5.

\section{OBSERVATIONS}

The observations of the fundamental rotational transitions of $\mathrm{NH}_{2} \mathrm{D}, \mathrm{NH}_{3}$, and $\mathrm{o}-\mathrm{H}_{2} \mathrm{O}$ in $\mathrm{L} 1689 \mathrm{~N}$ presented here ${ }^{12}$ were carried out in 2012 February-September using the Band 1 receivers of the Heterodyne Instrument for the Far-Infrared (HIFI; de Graauw et al. 2010) on the Herschel Space Observatory (Pilbratt et al. 2010). Both the WBS and HRS spectrometers were used in parallel. The WBS provides full coverage of the $4 \mathrm{GHz}$ intermediate frequency (IF) band in the upper and lower sidebands with a $1.1 \mathrm{MHz}$ resolution $\left(0.59 \mathrm{~km} \mathrm{~s}^{-1}\right.$ at $\left.557 \mathrm{GHz}\right)$, while the HRS provides higher spectral resolution spectra of selected lines of interest (spectral resolution of $0.12 \mathrm{MHz}$ or $0.07 \mathrm{~km} \mathrm{~s}^{-1}$ at $492 \mathrm{GHz}$ ). For mapping observations $\left(\sim 180^{\prime \prime} \times 190^{\prime \prime}\right.$ for water, $70^{\prime \prime} \times 90^{\prime \prime}$ for $\mathrm{NH}_{2} \mathrm{D}$ ), the HIFI OTF observing mode was used, while the frequency-switching mode was used for deeper, pointed

\footnotetext{
12 Herschel OBSIDs: 1342238591-38592, 38650, 50470, 51430; open time program Ammonia as a Tracer of the Earliest Stages of Star Formation, OT1_dlis_2, OT2_dlis_3.
} 
Table 1

Herschel, ACA, and CSO Observations of L1689N

\begin{tabular}{|c|c|c|c|}
\hline Species & $\begin{array}{l}\text { Frequency } \\
(\mathrm{GHz})\end{array}$ & Transition & $\begin{array}{c}E_{u} \\
(\mathrm{~K})\end{array}$ \\
\hline \multicolumn{4}{|c|}{ Herschel } \\
\hline $\mathrm{p}-\mathrm{NH}_{2} \mathrm{D}$ & 494.45455 & $1_{1,0}-0_{0,0}(0 \mathrm{a}-0 \mathrm{~s})$ & 23.7 \\
\hline $\mathrm{o}-\mathrm{H}_{2} \mathrm{O}$ & 556.93599 & $1_{1,0}-1_{0,1}$ & 61.0 \\
\hline $\mathrm{o}-\mathrm{NH}_{3}$ & 572.49816 & $1_{0}-0_{0}(0 \mathrm{~s}-0 \mathrm{a})$ & 27.5 \\
\hline SO & 558.08764 & $12_{13}-11_{12}$ & 194.4 \\
\hline SO & 559.31952 & $13_{13}-12_{12}$ & 201.1 \\
\hline SO & 560.17865 & $14_{13}-13_{12}$ & 192.7 \\
\hline \multicolumn{4}{|c|}{ ALMA Compact Array } \\
\hline $\mathrm{N}_{2} \mathrm{D}^{+}$ & 308.42227 & $4-3$ & 37.0 \\
\hline $\mathrm{CH}_{3} \mathrm{OH}$ & 309.29040 & $5_{1,4}-5_{0,5} \mathrm{~A}-+$ & 49.7 \\
\hline SO & 309.50244 & $2_{2}-2_{1}$ & 19.3 \\
\hline $\mathrm{ND}_{3}$ & 309.90949 & $1_{0}-0_{0}(0 \mathrm{a}-0 \mathrm{~s})$ & 14.9 \\
\hline Continuum & 309.963 & $\cdots$ & $\cdots$ \\
\hline Continuum & 353.949 & $\cdots$ & $\cdots$ \\
\hline \multicolumn{4}{|c|}{ Caltech Submillimeter Observatory } \\
\hline $\mathrm{DCO}^{+}$ & 216.11258 & $3-2$ & 10.4 \\
\hline $\mathrm{DCN}$ & 217.23854 & $3-2$ & 10.4 \\
\hline DNC & 228.91048 & $3-2$ & 11.0 \\
\hline $\mathrm{N}_{2} \mathrm{D}^{+}$ & 231.32183 & $3-2$ & 11.1 \\
\hline $\mathrm{HCN}$ & 265.88643 & $3-2$ & 12.8 \\
\hline $\mathrm{HCO}^{+}$ & 267.55763 & $3-2$ & 12.8 \\
\hline HNC & 271.98114 & $3-2$ & 13.1 \\
\hline $\mathrm{N}_{2} \mathrm{H}^{+}$ & 279.51175 & $3-2$ & 13.4 \\
\hline
\end{tabular}

observations. The data have been processed through the standard HIFI data reduction pipeline using HIPE version 10.0 (Ott 2010) and the resulting spectra were subsequently reduced using the GILDAS CLASS ${ }^{13}$ software package. The FWHM HIFI beam size is $\sim 44^{\prime \prime}$ at $492 \mathrm{GHz}$ and $38^{\prime \prime}$ at $557 \mathrm{GHz}$, and the main-beam efficiency is $76 \%$ (Roelfsema et al. 2012). The frequencies and quantum numbers of the transitions observed are listed in Table 1.

ALMA observations were obtained within two frequency settings (science goals): one near $310 \mathrm{GHz}$ and a second near $340 \mathrm{GHz}$. The spectral lines discussed in the present manuscript correspond to the $310 \mathrm{GHz}$ frequency setting and are summarized in Table 1 . A 10 pointing mosaic covering a $\sim 65^{\prime \prime} \times 80^{\prime \prime}$ region was observed on 7 occasions during 2014 January-April, using $117 \mathrm{~m}$ antennas of the Morita Array component of ALMA in Cycle 1. At each point of the mosaic, 15 minutes of data were collected for a sensitivity of $0.41 \mathrm{Jy} /$ beam in a $0.1 \mathrm{~km} \mathrm{~s}^{-1}$ channel. Four spectral windows were observed near $309 \mathrm{GHz}$, with varying spectral resolution. The $\mathrm{N}_{2} \mathrm{D}^{+} J=4-3$ line was in a $0.0625 \mathrm{GHz}$ width spectral window centered near $308.449 \mathrm{GHz}$ (velocity resolution of $0.045 \mathrm{~km} \mathrm{~s}^{-1}$ ), and the $\mathrm{ND}_{3}$ line lay in another $0.0625 \mathrm{GHz}$ width spectral window centered near $309.94 \mathrm{GHz}$. The $970 \mu \mathrm{m}$ dust continuum flux was extracted by averaging channels within two bands of $1.992 \mathrm{GHz}$ width centered near 308.053 and $309.873 \mathrm{GHz}$. The $\mathrm{CH}_{3} \mathrm{OH}$ and $\mathrm{H}_{3} \mathrm{O}^{+}$lines in the bands were flagged before averaging to avoid possible contamination. In the continuum image, the peak flux is $76 \mathrm{mJy} /$ beam and the integrated flux is $840 \mathrm{mJy}$. The rms in the image is $1.1 \mathrm{mJy} /$

\footnotetext{
${ }^{13}$ http://iram.fr/IRAMFR/GILDAS
}

beam (an excellent $\mathrm{S} / \mathrm{N}$ of 70 ) and the synthesized beam size is 5 ! $04 \times 3$ !" 16 at a position angle of $80^{\circ}$.

At $340 \mathrm{GHz}$, a 10 point mosaic covering the same $\sim 65^{\prime \prime} \times 80^{\prime \prime}$ region was observed on 6 occasions in 2014 April with the Morita Array component of ALMA with 15 minutes of on-source integration time per mosaic pointing. Eleven $7 \mathrm{~m}$ antennas were used for the observations with a maximum baseline of $50 \mathrm{~m}$. The $850 \mu \mathrm{m}$ dust continuum flux was extracted by averaging channels free of molecular line emission. The rms is $3.3 \mathrm{mJy}$, which is a factor of 3 higher than that in the $970 \mu \mathrm{m}$ continuum image, and the synthesized beam size is 4 !" $4 \times 2$ !" 9 at a position angle of $94^{\circ}$. The peak flux density of the $850 \mu \mathrm{m}$ dust continuum is $72 \mathrm{mJy} /$ beam and the integrated flux is $730 \mathrm{mJy}$. The $970 \mu \mathrm{m}$ continuum image thus has a much higher signal-to-noise ratio $(\mathrm{S} / \mathrm{N})$ and better preserves the low-level extended emission. Therefore, we use the $970 \mu \mathrm{m}$ data in the subsequent analysis.

The ALMA/NAASC staff performed bandpass calibration with the quasar J1733-1304 or J1427-4206, and the flux was calibrated with Titan. The phase calibrator was J1626-2951. The uncertainty in the absolute flux is $10 \%$. The calibrated visibilities were deconvolved and CLEANed with the CASA software package (version 4.2).

Single-dish observations of the $1 \mathrm{~mm}$ molecular transitions were carried out in 2013 May-June using the $10.4 \mathrm{~m}$ Leighton Telescope of the CSO on Mauna Kea, Hawaii. We used the wideband $230 \mathrm{GHz}$ facility SIS receiver and the FFTS backend that covers the full $4 \mathrm{GHz}$ IF range with a $270 \mathrm{kHz}$ channel spacing $\left(0.37 \mathrm{~km} \mathrm{~s}^{-1}\right.$ at $\left.220 \mathrm{GHz}\right)$. Pointing of the telescope was checked by performing five-point continuum scans of planets and strong dust continuum sources. The CSO mainbeam efficiency at $230 \mathrm{GHz}$ at the time of the observations was determined from total-power observations of planets to be $\sim 65 \%$. The absolute calibration uncertainty of the individual measurements is $\sim 15 \%$. The FWHM CSO beam size is $\sim 35^{\prime \prime}$ at $220 \mathrm{GHz}$, which is comparable to the Band 1 HIFI beam size.

\section{RESULTS}

\subsection{Herschel Space Observatory}

The overall morphology of the region, as observed with Herschel, is shown in Figure 1. The color image shows the integrated intensity of the $557 \mathrm{GHz}$ water emission between -15 and $25 \mathrm{~km} \mathrm{~s}^{-1}$. The location of IRAS 16293 is marked by the black contours of the integrated intensity of high-energy SO line emission, observed simultaneously, while the prestellar core is outlined by the white $\mathrm{NH}_{2} \mathrm{D}$ contours. There is an anticorrelation between the $\mathrm{NH}_{2} \mathrm{D}$ and water emission which surrounds and avoids the prestellar core. This can be seen even more clearly in the velocity channels maps (Figure 2) at blueshifted velocities. The strongest peak of the $\mathrm{H}_{2} \mathrm{O}$ emission is seen toward IRAS 16293. However, two secondary peaks are present to the northwest and southwest of the prestellar core. The blueshifted emission toward the northern peak extends to $\sim-8 \mathrm{~km} \mathrm{~s}^{-1}$, while the emission toward the south-western peak stops at $\sim-2 \mathrm{~km} \mathrm{~s}^{-1}$. Water is generally considered to be an excellent tracer of molecular outflows because water molecules are mostly frozen on dust grains in the cold gas and the spectra are less confused by foreground envelope absorption or emission compared to other tracers. We note that water vapor was detected in the isolated prestellar core L1544 in a very long HIFI integration (Caselli et al. 2012). A low gas-phase water 


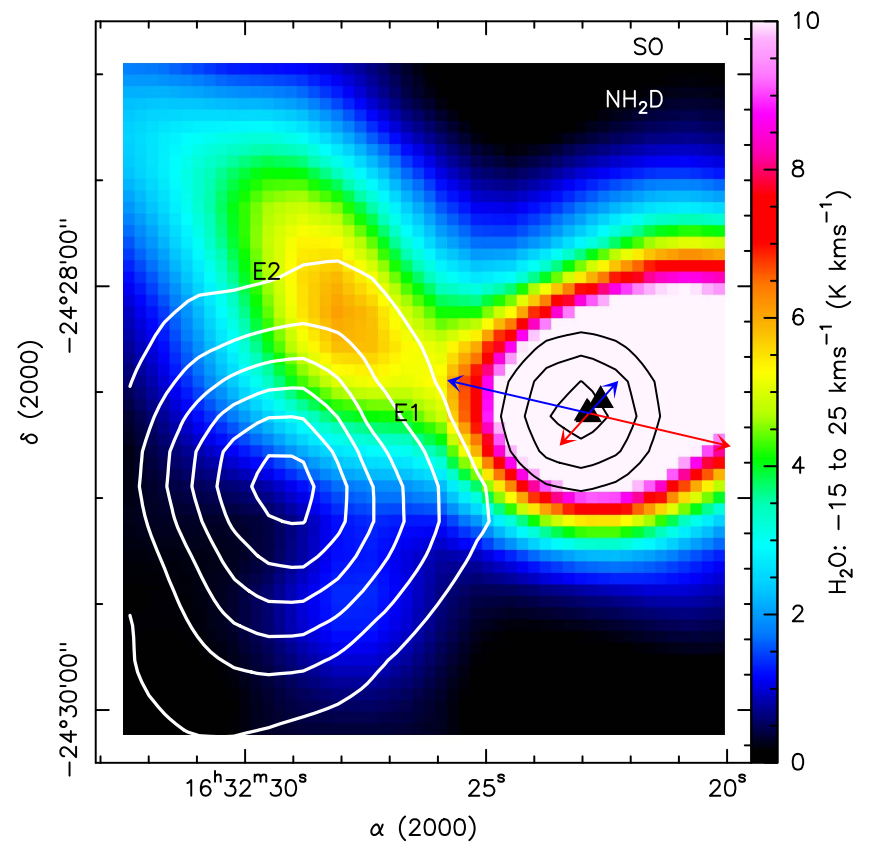

Figure 1. Color image of the integrated line intensity of the fundamental rotational transition of $\mathrm{o}-\mathrm{H}_{2} \mathrm{O}$ between -15 and $25 \mathrm{~km} \mathrm{~s}^{-1}$, with overlaid white contours of the integrated intensity of the fundamental rotational transition of $\mathrm{NH}_{2} \mathrm{D}$ between 1.7 and $5.5 \mathrm{~km} \mathrm{~s}^{-1}$ (including the 3 hyperfine components). The $\mathrm{NH}_{2} \mathrm{D}$ emission shows the location of the prestellar core, while the location of the solar-type protostar IRAS 16293 driving the molecular outflow is shown by the black contours of the excited SO emission (average of the three transitions listed in Table 1). Contour levels for $\mathrm{NH}_{2} \mathrm{D}$ are from $20 \%$ to $95 \%$ of the peak $\left(0.65 \mathrm{~K} \mathrm{~km} \mathrm{~s}^{-1}\right)$ with a step of $15 \%$. For SO, the contour levels are $50 \%, 70 \%$, and $90 \%$ of the peak $\left(2.25 \mathrm{~K} \mathrm{~km} \mathrm{~s}^{-1}\right)$. Black triangles mark the locations of IRAS 16293 A and B (lower and upper, respectively). The red and blue arrows mark the directions of the compact $\mathrm{CO}$ outflows observed with the SMA (Girart et al. 2014), which have opposite polarity to the large-scale outflows seen in earlier single-dish studies (e.g., Stark et al. 2004). E1 and E2 mark the locations of the two SiO clumps of Hirano et al. (2001).

abundance in this source is maintained by UV photons locally produced by the interaction of $\mathrm{H}_{2}$ molecules with galactic cosmic rays.

A high-resolution spectrum of the $\mathrm{NH}_{2} \mathrm{D}$ line toward the prestellar core is shown in Figure 3 (upper panel). The hyperfine structure (HFS) is well resolved spectrally, given the narrow line width of $0.38 \mathrm{~km} \mathrm{~s}^{-1}$. The HFS fit parameters (the line center velocity and width, the excitation temperature, and the line center optical depth) are given in Table 2 . The weakest hyperfine component is optically thin $(\tau \sim 0.5)$. The spectrum of $\mathrm{NH}_{3}$ (lower panel) looks very peculiar, as only the weakest HFS component is clearly seen, with an intensity lower than that of the weakest $\mathrm{NH}_{2} \mathrm{D}$ hyperfine component. The $\mathrm{NH}_{3}$ emission is clearly sub-thermally excited, and the resulting spectrum is affected by the strong temperature and density gradients along the line of sight. A likely explanation of the peculiar line shape is that the two strongest $\mathrm{NH}_{3}$ hyperfine components are almost completely absorbed by the foreground gas. The $\mathrm{NH}_{3}$ spectrum toward IRAS 16293 shows absorption at $\sim 3.85 \mathrm{~km} \mathrm{~s}^{-1}$ (red vertical lines in Figure 3, lower panel). Since the molecular emission toward the prestellar core is blueshifted with respect to the envelope velocity, the weakest blueshifted $\mathrm{NH}_{3}$ hyperfine component is much less affected by foreground absorption. An alternative explanation that the strongest $\mathrm{NH}_{3}$ emission feature corresponds to the main HFS component at a velocity of $\sim 2.5 \mathrm{~km} \mathrm{~s}^{-1}$, and that the weakest
HFS component is simply not detected, can be discarded as there are no other molecular tracers emitting at $2.5 \mathrm{~km} \mathrm{~s}^{-1}$ in this source. Due to the contamination by the foreground absorption, the optical depth of the $\mathrm{NH}_{3}$ line cannot be directly determined from the HFS fit, and thus the line is not useful for quantitative determination of the molecular column density and the isotopic $\mathrm{D} / \mathrm{H}$ ratio.

Figure 4 shows the integrated line intensity of the weakest $\mathrm{NH}_{3}$ HFS component with overlaid contours of the $\mathrm{NH}_{2} \mathrm{D}$ and $\mathrm{SO}$ emission. In the vicinity of the prestellar core, $\mathrm{NH}_{3}$ peaks $\sim 10^{\prime \prime}$ to the east of $\mathrm{NH}_{2} \mathrm{D}$. The offset may be indicative of an abundance gradient between the two ammonia isotopologues. However, given the high opacity of the $\mathrm{NH}_{3}$ line, it may simply be due to radiative transfer effects.

An interaction with the outflow should have an effect on the velocity field of the prestellar core. Figure 5 shows the $\mathrm{NH}_{2} \mathrm{D}$ line center velocity and width as a function of position (top left and right panels, respectively). There is a clear trend of the line velocity and width increasing from the northeast to the west/ southwest. The $\mathrm{NH}_{2} \mathrm{D}$ spectra at three selected positions, shown in the bottom row, display this transition from a quiescent gas with a narrow line width in the northeast to a much more perturbed gas in the western/south-western part of the core.

\subsection{ALMA Compact Array}

To further investigate the morphology of the prestellar core, we have imaged the $970 \mu \mathrm{m}$ dust continuum emission together with a number of molecular tracers, including deuterated species, using the ACA. The $\mathrm{N}_{2} \mathrm{D}^{+}$emission (Figure 6, color image and white contours) extends south/southwest from the peak of the $\mathrm{NH}_{2} \mathrm{D}$ emission, as observed with HIFI (blue star), and peaks $\sim 5^{\prime \prime}$ to the north with respect to the dust continuum (black contours, left panel). While the northern tip of the $\mathrm{N}_{2} \mathrm{D}^{+}$emission coincides with the $\mathrm{NH}_{2} \mathrm{D}$ peak, the very large $44^{\prime \prime}$ HIFI beam prevents a detailed comparison of the morphology on the angular scales probed by the ACA. We note, however, that the dust emission terminates rather abruptly in this part of the core, with the molecular emission extending further to the north. The $\mathrm{ND}_{3}$ emission (black contours, right panel) more closely resembles $\mathrm{N}_{2} \mathrm{D}^{+}$than the dust continuum emission, although the $\mathrm{ND}_{3}$ emission peak is displaced by $\sim 2$ !" 5 east with respect to $\mathrm{N}_{2} \mathrm{D}^{+}$(possibly due to optical depth effects, as the $\mathrm{N}_{2} \mathrm{D}^{+}$line has a significant opacity; see Table 2).

Figure 7 shows $\mathrm{N}_{2} \mathrm{D}^{+}$and $\mathrm{ND}_{3}$ spectra toward the respective emission peaks with single velocity component HFS fits overlaid in red. Vertical green lines mark the velocities and relative intensities of the HFS components. The HFS fit parameters are given in Table 2. The $\mathrm{N}_{2} \mathrm{D}^{+}$emission is quite optically thick and does not directly trace the molecular column density. The derived excitation temperature, which can be taken as a lower limit of the gas kinetic temperature (the emission does not have to be thermalized), is significantly lower that the isothermal dust temperature of $16 \mathrm{~K}$ derived from a spectral energy distribution fit by Stark et al. (2004). However, these authors point out that a cold, high-density region with $T_{d} \ll 16 \mathrm{~K}$ can easily be hidden at the center of the core. In fact, in a recent study, Bacmann et al. (2016) derive a temperature profile increasing from $11 \mathrm{~K}$ at the core center to $16 \mathrm{~K}$ in the outer envelope. Even lower dust temperatures, in the range of $8-16 \mathrm{~K}$, are derived by Pagani et al. (2016). 


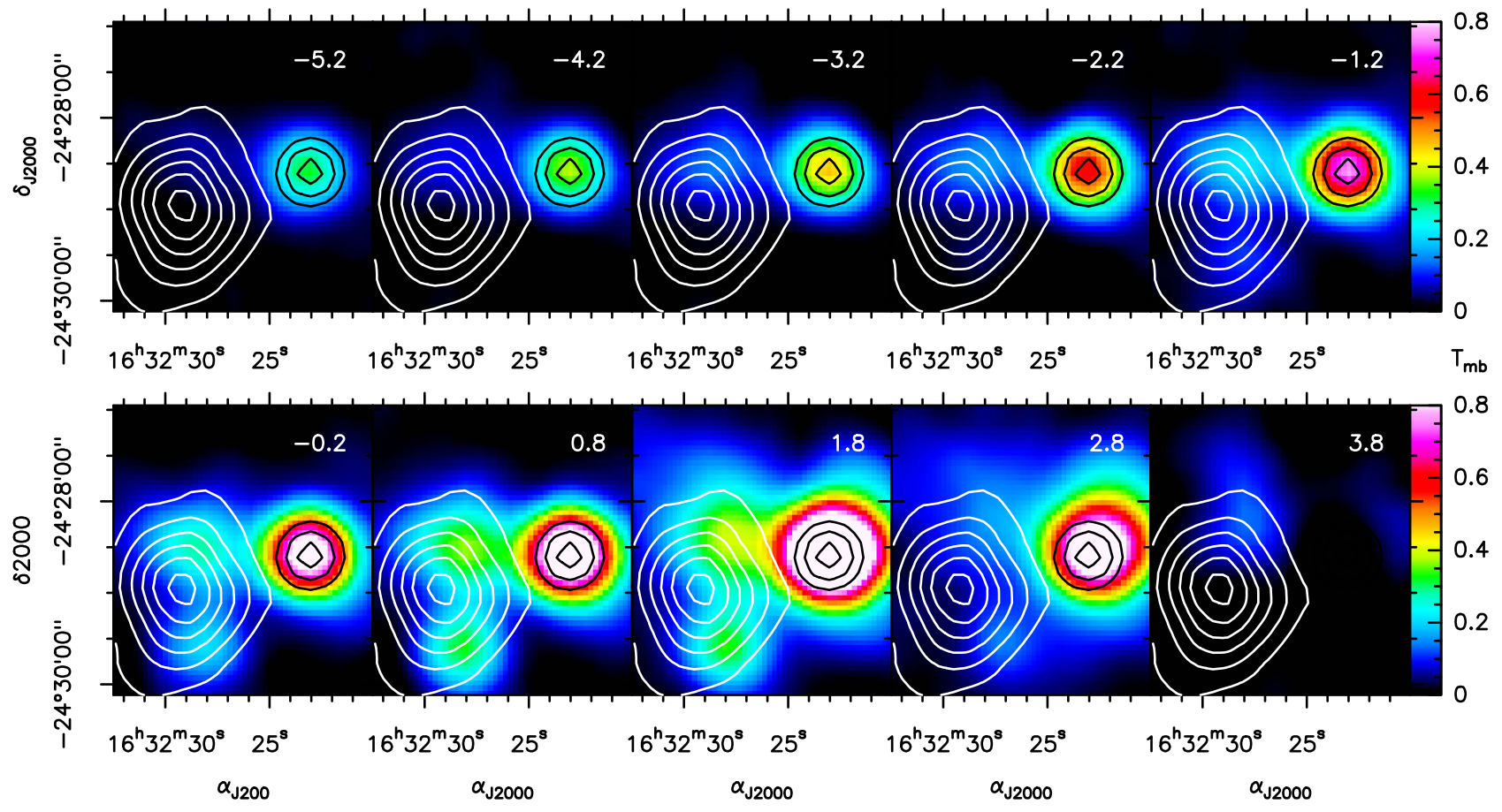

Figure 2. Channel maps of the blueshifted $557 \mathrm{GHz}$ water emission, between -5.2 and $3.8 \mathrm{~km} \mathrm{~s}^{-1}$ with overlaid white contours of the $\mathrm{NH}_{2} \mathrm{D}$ integrated line intensity between 1.7 and $5.5 \mathrm{~km} \mathrm{~s}^{-1}$ showing the location of the prestellar core and black contours of the excited SO emission showing the location of the protostar IRAS 16293. Contour levels are the same as in Figure 1.

The $\mathrm{ND}_{3}$ emission is optically thin. A HFS fit gives a slightly larger line width compared to $\mathrm{N}_{2} \mathrm{D}^{+}$(Table 2), which however is consistent with that of the $\mathrm{NH}_{2} \mathrm{D}$ emission in the ten times larger HIFI beam. We thus see no evidence in our deuterated ammonia data for the turbulent velocity to vary with radius, as seen in some sources. The complex HFS of $\mathrm{N}_{2} \mathrm{D}^{+}$and a limited $\mathrm{S} / \mathrm{N}$ of the $\mathrm{ND}_{3}$ spectrum prevent us from drawing any conclusions about the presence of possible infall motions in the core. The $\sim 0.4 \mathrm{~km} \mathrm{~s}^{-1}$ FWHM line width roughly corresponds to the $\mathrm{H}_{2}$ thermal line width at $7 \mathrm{~K}$ and is 3-4 times larger than the expected thermal line width of $\mathrm{ND}_{3}, \mathrm{NH}_{2} \mathrm{D}$, or $\mathrm{N}_{2} \mathrm{D}^{+}$. This shows that the line broadening is mainly non-thermal and that sonic or somewhat sub-sonic turbulent motions are dominant even in the northern, quiescent part of the core. This is different from the typical prestellar cores in Taurus where line widths are essentially thermal and is perhaps related to the interaction with the outflow.

Figure 8 shows the distribution of the $\mathrm{CH}_{3} \mathrm{OH}$ and $\mathrm{SO}$ emission in the vicinity of the prestellar core (blue contours, left and right panels, respectively). The background image is the blueshifted $\mathrm{H}_{2} \mathrm{O}$ emission observed with HIFI, while the black contours show the $970 \mu \mathrm{m}$ dust continuum emission. A significant fraction of the $\mathrm{CH}_{3} \mathrm{OH}$ and $\mathrm{SO}$ emission $(\sim 80 \%$ $85 \%)$ is resolved out in the ACA images. However, we see strong methanol emission associated with the two blueshifted water peaks to the northwest and southwest of the prestellar core, while the SO emission is only associated with the weaker south-western blueshifted water peak. Pagani et al. (2016) report the presence of a new outflow which could be responsible for the emission peak southwest of the prestellar core. No SO or methanol emission is detected toward the prestellar core.

\subsection{Caltech Submillimeter Observatory}

To study further the large-scale morphology and kinematics of the molecular gas in the vicinity of the prestellar core, we have mapped the $1 \mathrm{~mm}$ lines of $\mathrm{DCO}^{+}, \mathrm{DCN}, \mathrm{DNC}$, and $\mathrm{N}_{2} \mathrm{D}^{+}$, as well as their hydrogenated counterparts $\mathrm{HCO}^{+}$, $\mathrm{HCN}, \mathrm{HNC}$, and $\mathrm{N}_{2} \mathrm{H}^{+}$, using the CSO (Table 1). ${ }^{14}$ The resulting maps of deuterated species are shown as color images in Figure 9. Although all deuterated species peak in the general vicinity of the prestellar core, significant differences among their morphologies can be seen. The prestellar core, as traced by the $\mathrm{NH}_{2} \mathrm{D}$ emission, is best seen in the DNC and $\mathrm{N}_{2} \mathrm{D}^{+}$images. The $\mathrm{DCO}^{+}$emission, being the brightest and thus most suitable for mapping of extended areas, is not confined to the immediate vicinity of the prestellar core and shows a clear extension toward the E1 shocked region, while DCN peaks in between the prestellar core and E1. This is not surprising, as this molecule has been suggested to be produced by "warm chemistry" driven by $\mathrm{CH}_{2} \mathrm{D}^{+}$in the gas with temperatures of the order of $50 \mathrm{~K}$, where the $\mathrm{H}_{2} \mathrm{D}^{+}$driven deuteration reactions are already suppressed (Roueff et al. 2007; Parise et al. 2009). We note that, similar to $\mathrm{NH}_{2} \mathrm{D}$, the single-dish $\mathrm{N}_{2} \mathrm{D}^{+}$and $\mathrm{DNC}$ emission also peaks to the north of the ACA $\mathrm{N}_{2} \mathrm{D}^{+}$and $\mathrm{ND}_{3}$ sources. Among the hydrogenated molecular tracers, only $\mathrm{N}_{2} \mathrm{H}^{+}$emission reveals the prestellar core, peaking close to the location of the ACA $\mathrm{N}_{2} \mathrm{D}^{+}$and $\mathrm{ND}_{3}$ sources. All of the other tracers largely follow the distribution of the blueshifted water emission, peaking toward the E1 source with a secondary peak to the southwest (labeled HE2 by Castets et al. 2001).

\footnotetext{
${ }^{14}$ The $\mathrm{N}_{2} \mathrm{H}^{+}$data have previously been used in the models of Daniel et al. (2016a) to derive the nitrogen isotopic ratio in L1689N.
} 


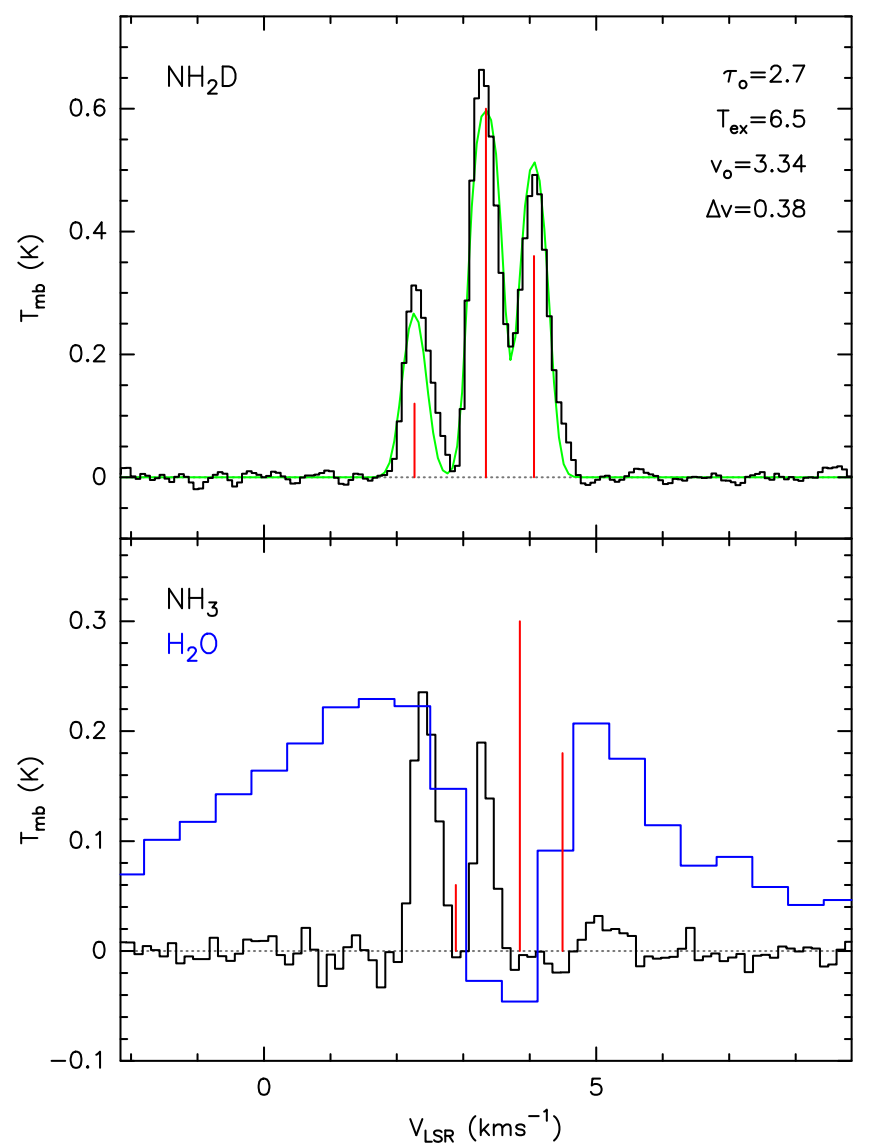

Figure 3. Spectra of the $\mathrm{NH}_{2} \mathrm{D}$ and $\mathrm{NH}_{3}$ lines toward the prestellar core $\left(\alpha_{J 2000}=16^{\mathrm{h}} 32^{\mathrm{m}} 29^{\mathrm{s}} .40, \delta_{J 2000}=-24^{\circ} 28^{\prime} 53^{\prime \prime}\right)$ taken with the HRS spectrometer. The green line in the upper panel is the HFS fit, which gives the line center optical depth of $2.7 \pm 0.07$ and the excitation temperature of $6.5 \pm$ $0.8 \mathrm{~K}$. The $\mathrm{H}_{2} \mathrm{O}$ spectrum observed with the WBS is shown in blue in the lower panel. Red vertical lines marked the velocities of the $\mathrm{NH}_{2} \mathrm{D}$ and $\mathrm{NH}_{3}$ HFS components, with the relative line intensities corresponding to optically thin LTE emission (5:3:1). The $\mathrm{NH}_{3}$ HFS components are plotted with respect to the velocity of the foreground cloud envelope at $3.85 \mathrm{~km} \mathrm{~s}^{-1}$, as determined from the $\mathrm{NH}_{3}$ absorption spectrum toward IRAS 16293 .

Table 2

Results of the HFS Fits

\begin{tabular}{lcccc}
\hline \hline Line & $\begin{array}{c}v_{0} \\
\left(\mathrm{~km} \mathrm{~s}^{-1}\right)\end{array}$ & $\begin{array}{c}\Delta v \\
\left(\mathrm{~km} \mathrm{~s}^{-1}\right)\end{array}$ & $\begin{array}{l}T_{\text {ex }} \\
(\mathrm{K})\end{array}$ & $\tau_{0}$ \\
\hline $\mathrm{NH}_{2} \mathrm{D}$ & 3.34 & 0.38 & 6.5 & 2.7 \\
$\mathrm{ND}_{3}$ & 3.45 & 0.40 & 6.5 & 0.43 \\
$\mathrm{~N}_{2} \mathrm{D}^{+}$ & 3.68 & 0.34 & 7.9 & 3.3 \\
\hline
\end{tabular}

Note. Formal $1 \sigma$ fit uncertainties provided by CLASS are $\sim 0.002 \mathrm{~km} \mathrm{~s}^{-1}$ for the line velocity and width, and $\sim 2.5 \%$ for the line center optical depth.

\section{DISCUSSION}

\subsection{Properties of the Dust Continuum Source}

The $970 \mu \mathrm{m}$ continuum source detected with ACA is elongated approximately in the north-south direction. A twodimensional Gaussian fit gives a FWHM source size of 13 ! $2 \times 8$ !" 6 at a position ange of $2^{\circ}$. After deconvolving the beam, the intrinsic source size is 12 !" $9 \times 7$ !" 0 , or $1540 \times 840 \mathrm{au}$, assuming a distance of $120 \mathrm{pc}$ (Loinard et al. 2008). The column density profile of the core is well described by a two-dimensional Gaussian. However, the spatial

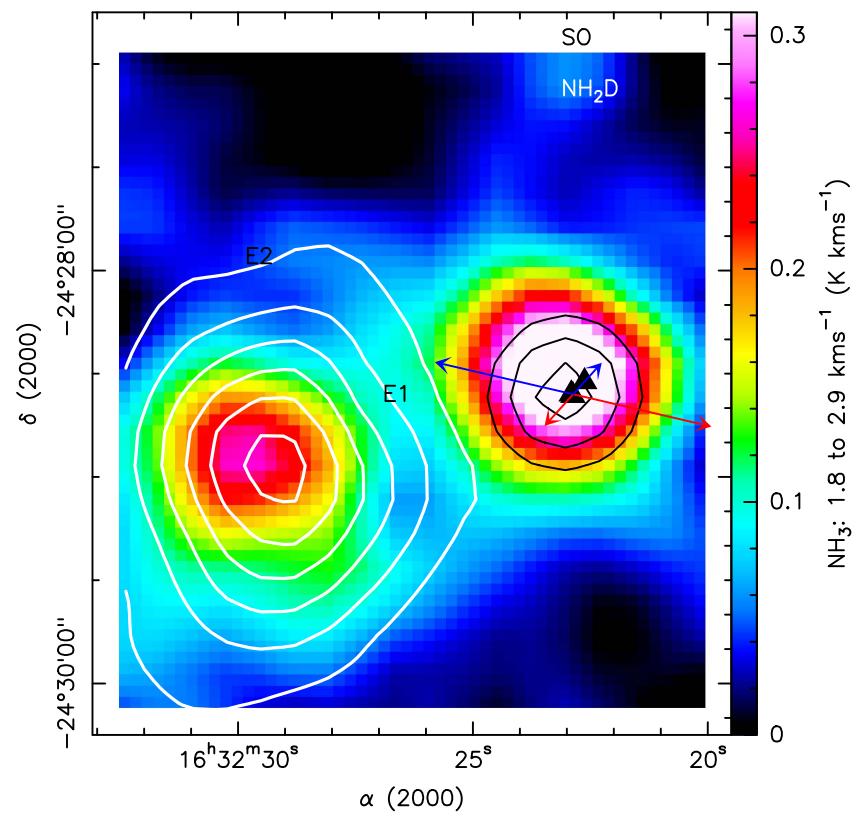

Figure 4. Color image of the integrated line intensity of the weakest $\mathrm{NH}_{3} \mathrm{HFS}$ component between -0.8 and $1.9 \mathrm{~km} \mathrm{~s}^{-1}$ with overlaid contours of $\mathrm{NH}_{2} \mathrm{D}$ and SO (white and black, respectively). Contour levels and symbols are the same as in Figure 1.

dynamical range in the current image is rather low: only 3.5. Higher angular resolution observations with the main ALMA array will thus be required to study this important aspect.

The peak $970 \mu \mathrm{m}$ continuum flux is $76.0 \mathrm{mJy} /$ beam and the integrated flux is $0.84 \mathrm{Jy}$. To estimate the $\mathrm{H}_{2}$ column density and the mass of the compact dust source, we scale the grain opacity coefficient $\kappa_{1.3 \mathrm{~mm}}=0.005 \mathrm{~cm}^{2} \mathrm{~g}^{-1}$ (Motte et al. 1998), which is appropriate for dense prestellar clumps, with a $\nu^{2}$ frequency dependence. This leads to $\kappa_{970 \mu \mathrm{m}}=$ $0.009 \mathrm{~cm}^{2} \mathrm{~g}^{-1} .15$ For a $16 \mathrm{~K}$ dust temperature (Stark et al. 2004), we derive an $\mathrm{H}_{2}$ column density of $1.8 \times$ $10^{23} \mathrm{~cm}^{-2}$. However, if the dust temperature is $11 \mathrm{~K}$, as suggested by Bacmann et al. (2016), then the corresponding column density increases to $3.3 \times 10^{23} \mathrm{~cm}^{-2}$. Even in the latter case, the dust emission is optically thin (a $970 \mu \mathrm{m}$ optical depth of $\sim 0.01)$. Assuming a $\nu^{2}$ frequency dependence, the dust opacity at $557 \mathrm{GHz}$ is also low. Therefore, the observed anticorrelation between water and $\mathrm{NH}_{2} \mathrm{D}$ emission, as seen by HIFI, cannot be explained by foreground dust absorption.

Assuming a line-of-sight source size of 9".5 or $1140 \mathrm{au}$, equal to the geometric mean of the FWHM sizes in the plane of the sky, we derive an $\mathrm{H}_{2}$ volume density of (1.1-1.9) $\times$ $10^{7} \mathrm{~cm}^{-3}$, depending on the dust temperature. This is an order of magnitude higher than the values derived in earlier singledish studies (Stark et al. 2004; Vastel et al. 2012), but is consistent with the best-model value of $1.4 \times 10^{7} \mathrm{~cm}^{-2}$ in the recent study of Bacmann et al. (2016). The high central density in the model of Bacmann et al. (2016) is in fact directly related to the presence of a temperature gradient. The best-fit powerlaw density exponent in these latest models is 1.7 , as compared to the low value of $\sim 1$ in the isothermal model of Stark et al.

\footnotetext{
15 Stark et al. (2004) used a grain opacity coefficient of $\kappa_{1.2 \mathrm{THz}}=0.1 \mathrm{~cm}^{-2} \mathrm{~g}^{-1}$ When extrapolated with a $\nu^{2}$ frequency dependence, this gives $\kappa_{870 \mu \mathrm{m}}=$ $0.008 \mathrm{~cm}^{2} \mathrm{~g}^{-1}$, which is about $25 \%$ lower than the value used here.
} 

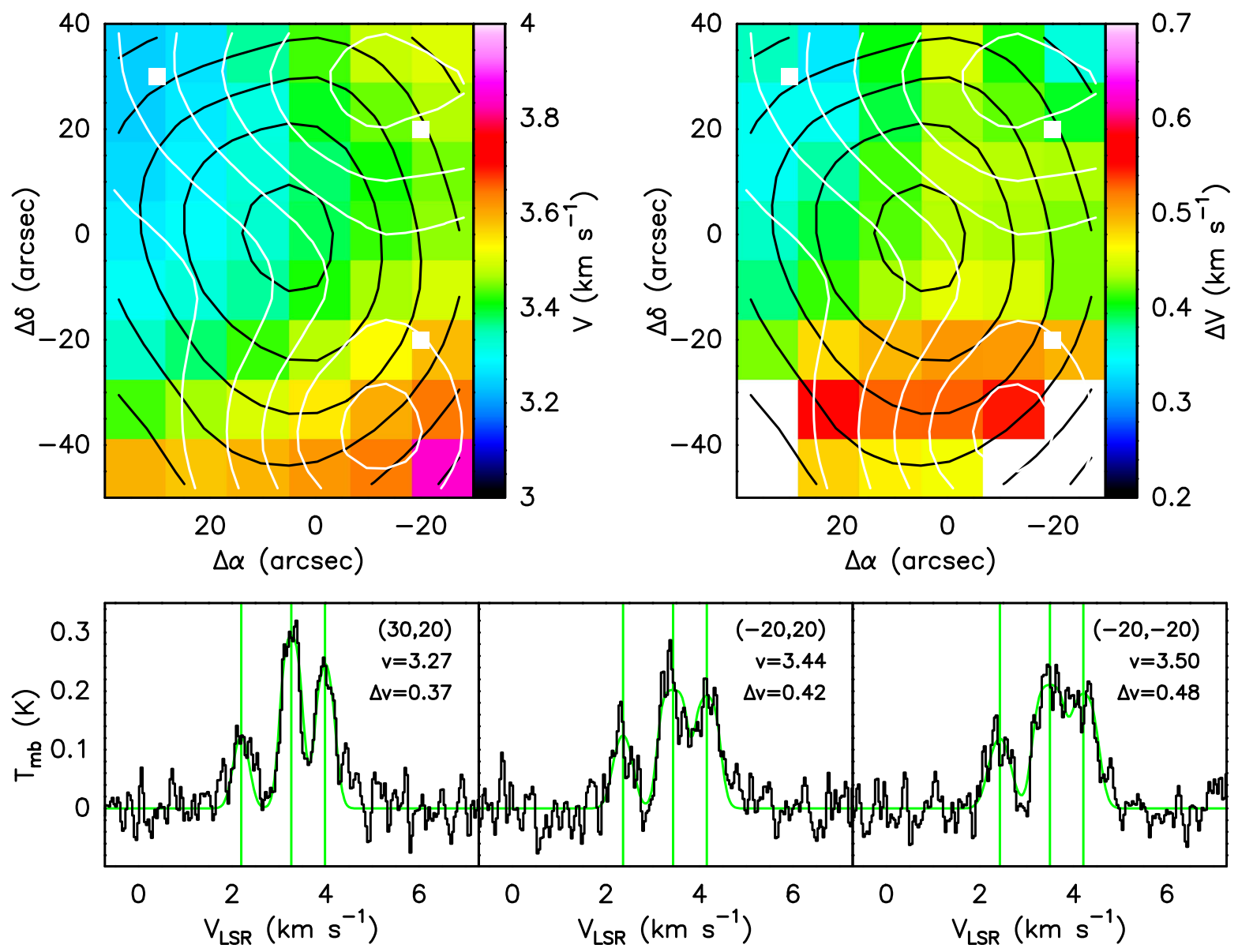

Figure 5. (Top) Maps of the $\mathrm{NH}_{2} \mathrm{D}$ line velocity and width across the prestellar core (left and right, respectively). Black contours show the $\mathrm{NH}_{2} \mathrm{D}$ integrated line intensity and white contours blueshifted $\mathrm{H}_{2} \mathrm{O}$ emission between -0.2 and $3.8 \mathrm{~km} \mathrm{~s}^{-1}$. Contour levels are from $20 \%$ to $95 \%$ of the peak $\left(0.65\right.$ and $1.11 \mathrm{~K}$ km s${ }^{-1}$ for $\mathrm{NH}_{2} \mathrm{D}$ and $\mathrm{H}_{2} \mathrm{O}$, respectively), with a step of $15 \%$. The $(0,0)$ position corresponds to $\alpha_{J 2000}=16^{\mathrm{h}} 32^{\mathrm{m}} 28^{\mathrm{s}} .84, \delta_{J 2000}=-24^{\circ} 28^{\prime} 57^{\prime \prime}$. $(\mathrm{Bottom}) \mathrm{NH}_{2} \mathrm{D}$ spectra at three positions across the prestellar core, marked by white squares in the top row, demonstrating the change in the line velocity and width from the northeast to the southwest. The spectra have been convolved with a $25^{\prime \prime}$ Gaussian to improve the $\mathrm{S} / \mathrm{N}$.

(2004). The prestellar core can also be identified with source 84 (SMM 19) of Pattle et al. (2015), who derive a temperature of $11.8 \mathrm{~K}$ and a density of $3.3 \times 10^{7} \mathrm{~cm}^{-3}$. The new single-dish and interferometric data presented here provide additional constraints for detailed radiative transfer modeling, which will be the subject of a separate study.

The total mass of the compact continuum source seen in the ACA image is $0.22 M_{\odot}$, assuming a dust temperature of $16 \mathrm{~K}$ $\left(0.42 M_{\odot}\right.$ for a dust temperature of $\left.11 \mathrm{~K}\right)$, a small fraction of the total mass of the prestellar core $2.45 M_{\odot}$ (Stark et al. 2004; assuming a $16 \mathrm{~K}$ dust temperature and scaling to the distance of $120 \mathrm{pc}$ used here). Pattle et al. (2015) derive a mass of 0.30 $M_{\odot}$ (after scaling to the distance of $120 \mathrm{pc}$ ). This source thus has a mass characteristic of the Very Low Luminosity Objects (VeLLOs) detected by Spitzer (see, e.g., Dunham et al. 2014 and references therein), which have bolometric luminosities below $0.1 L_{\odot}$ (Di francesco et al. 2007). Stark et al. (2004) derive a total bolometric luminosity of $1.3 L_{\odot}$ toward the prestellar core in L1689N, after scaling to the distance of $120 \mathrm{pc}$. However, it is not clear what fraction of this luminosity is associated with the compact continuum source detected in the ACA data, as Spitzer IRAC images do not reveal any $4.5 \mu \mathrm{m}$ continuum sources at this location (Pagani et al. 2016).

\subsection{Interaction with the Outflow}

The environment of L1689N shows a complex morphology. Optically thick molecular lines show a pronounced self-reversal at the systemic velocity of $\sim 3.8 \mathrm{~km} \mathrm{~s}^{-1}$. The parent molecular cloud has a mean density of (2-3) $\times 10^{4} \mathrm{~cm}^{-3}$ and a kinetic temperature of $12 \mathrm{~K}$ based on ammonia observations (Menten et al. 1987). Multiple large-scale molecular outflows are present, driven by IRAS 16293 (Mizuno et al. 1990; Lis et al. 2002a; Stark et al. 2004). The SMA observations of Yeh et al. (2008) imply a primarily east-west outflow with blueshifted emission to the west. These authors suggest that this small-scale outflow may be the inner part of the large-scale east-west outflow. More recent SMA data (Girart et al. 2014) suggest that the $\mathrm{CO}$ emission at moderately high velocities arises from two bipolar outflows, which appear perpendicular to each other and both originate from IRAS 16293A. The more compact outflow is impacting the circumstellar gas around component $\mathrm{B}$, and is possibly being redirected. The directions of the two compact outflows, as indicated by the SMA observations of Girart et al. (2014), are marked in Figure 1.

The outflow originates in the two sources at the center of IRAS 16293A: A-E and A-W. In fact, VLA observations reveal that the continuum source A-E orbits A-W, which had a recent continuum ejection event (Loinard et al. 2007). That event defined the flow originating in A-W with the redshifted 


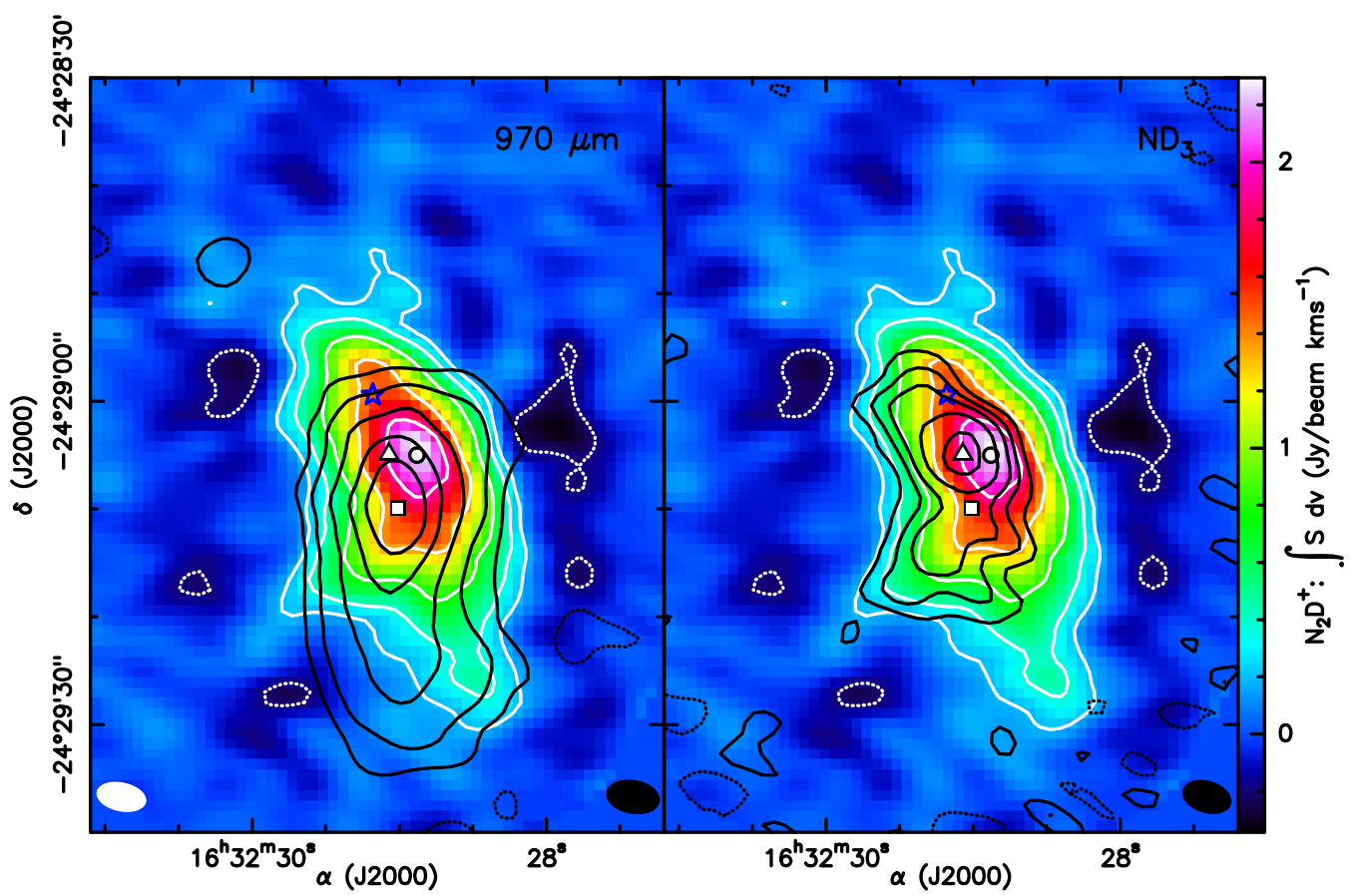

Figure 6. Integrated line intensity of the $\mathrm{N}_{2} \mathrm{D}^{+}$emission (color image and white contours) with overlaid black contours of the $970 \mu \mathrm{m}$ continuum emission (left panel) and integrated line intensity of the $\mathrm{ND}_{3}$ emission (right panel). $\mathrm{N}_{2} \mathrm{D}^{+}$emission has been integrated over velocities 3.04-4.17 and 5.24-5.77 km s${ }^{-1}$, including two groups of the strongest hyperfine components, while the $\mathrm{ND}_{3}$ emission has been integrated over velocities 3.08-3.79 and $4.32-4.97 \mathrm{~km} \mathrm{~s}{ }^{-1}$, including the two strongest hyperfine components (including the weaker hyperfine components does not improve the $\mathrm{S} / \mathrm{N}$ in the resulting images). Contour levels for $\mathrm{N}_{2} \mathrm{D}^{+}$and the continuum emission are $-5,5,10,20,35$, and 50 times the rms $\left(3.84 \times 10^{-2} \mathrm{Jy} /\right.$ beam km s${ }^{-1}$ and $1.11 \times 10^{-3} \mathrm{Jy} /$ beam, respectively). For ND 3 , contour levels are $3,3,5,7,10$, and 14 times the rms $\left(3.67 \times 10^{-2} \mathrm{Jy} /\right.$ beam $\left.\mathrm{km} \mathrm{s}^{-1}\right)$. The synthesized beams are $4 ! \prime 71 \times 22^{\prime \prime} 69$ at $78^{\circ}$ for $\mathrm{N}_{2} \mathrm{D}^{+}$(shown in the lower left corner of the left panel), $4^{\prime \prime} 70 \times 3^{\prime \prime}, 00$ at $73^{\circ}$ for $\mathrm{ND}_{3}$ (lower right corner of the right panel), and $5^{\prime \prime} 04 \times 3^{\prime \prime} 16$ at $80^{\circ}$ for $970 \mu \mathrm{m}$ continuum (lower right corner of the left panel). Symbols mark the location of the emission peaks: $\mathrm{N}_{2} \mathrm{D}^{+}$(circle; $16^{\mathrm{h}} 32^{\mathrm{m}} 28^{\mathrm{s}} \mathrm{s} 8,-24^{\circ} 29^{\prime} 05^{\prime \prime}$ ) ), $\mathrm{ND}_{3}$ (triangle; $16^{\mathrm{h}} 32^{\mathrm{m}} 29^{\mathrm{s}} .07,-24^{\circ} 29^{\prime} 04^{\prime \prime}$ ) ), continuum (square; $16^{\mathrm{h}} 32^{\mathrm{m}} 29^{\mathrm{s}} .01,-24^{\circ} 29^{\prime} 09^{\prime \prime} \cdot 9$ ), and $\mathrm{NH}_{2} \mathrm{D}$ (star; $\left.16^{\mathrm{h}} 32^{\mathrm{m}} 29^{\mathrm{s}} .2-24^{\circ} 28^{\prime} 59^{\prime \prime} .4\right)$.
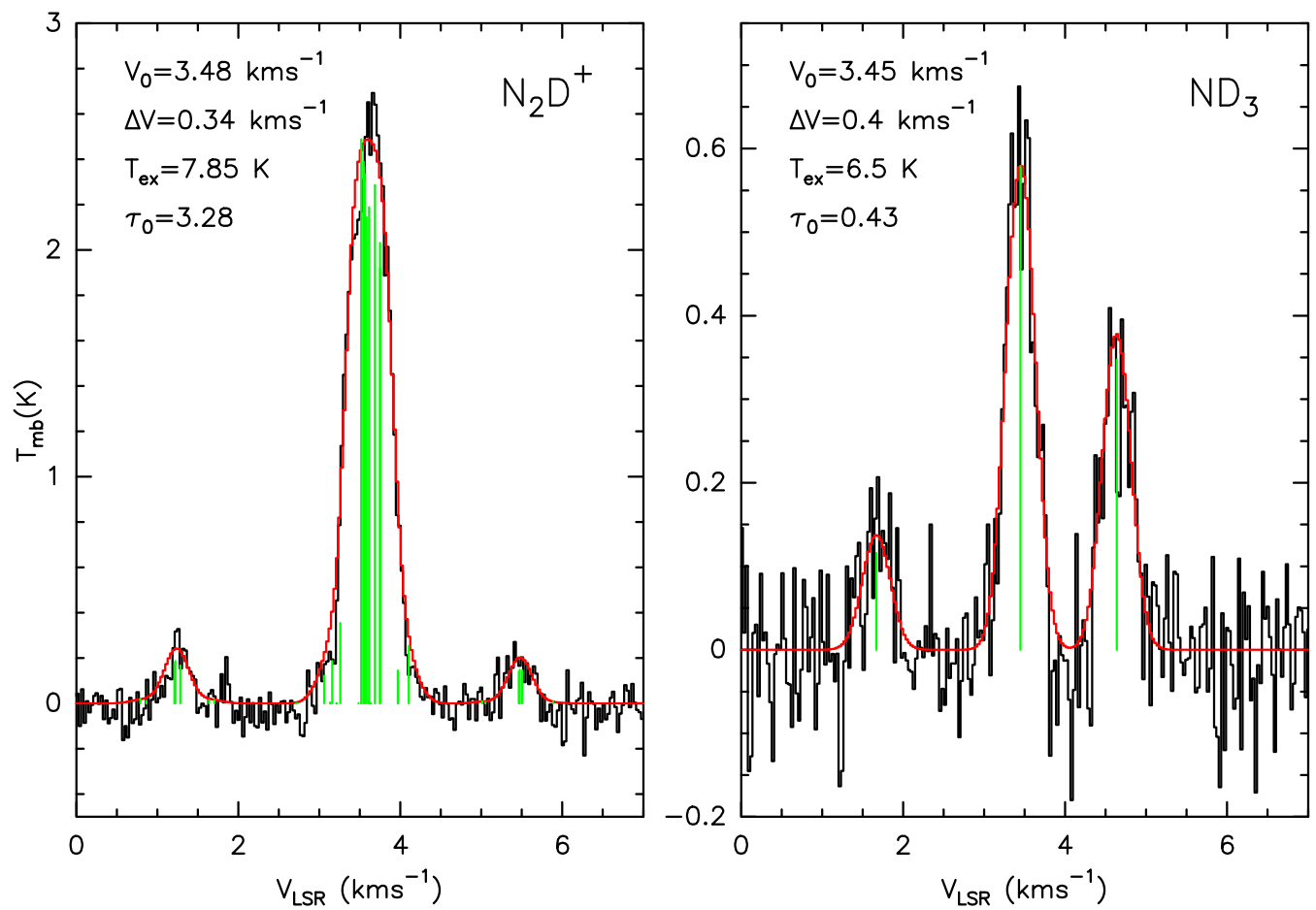

Figure 7. $\mathrm{N}_{2} \mathrm{D}^{+}$and $\mathrm{ND}_{3}$ spectra (left and right panels, respectively) toward the respective emission peaks. HFS fits are shown in red, with the best-fit parameters shown in the upper left corners. For $\mathrm{N}_{2} \mathrm{D}^{+}$the LSR velocity scale is plotted with respect to the CDMS frequency of $308.422 .27 \mathrm{GHz}$. 


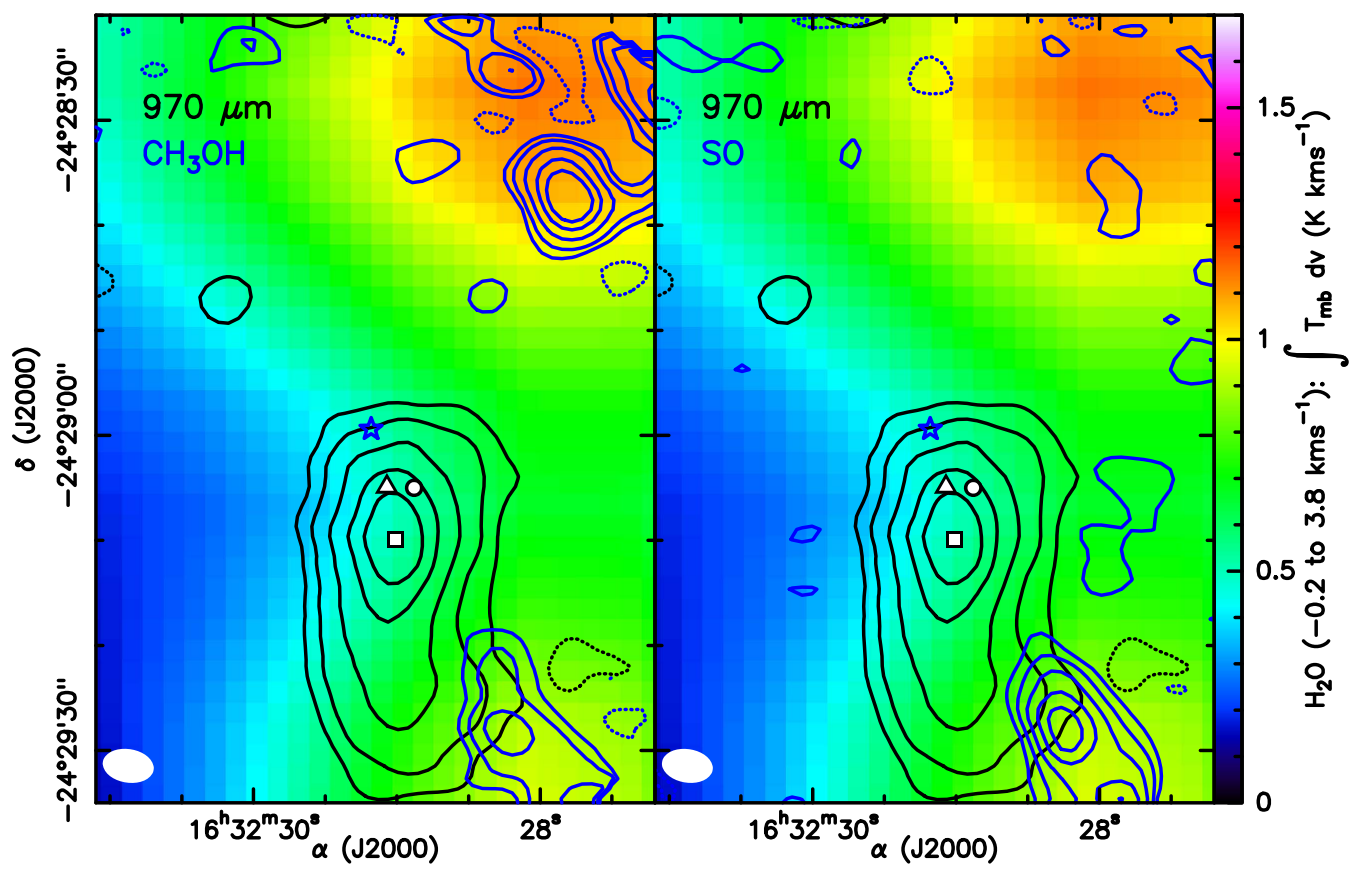

Figure 8. Integrated line intensity of the blueshifted $\mathrm{H}_{2} \mathrm{O}$ emission between -0.2 and $3.8 \mathrm{~km} \mathrm{~s}^{-1}$ (color image) with overlaid black contours of the $970 \mu \mathrm{m}$ continuum emission and blue contours of the integrated line intensity of the $\mathrm{CH}_{3} \mathrm{OH}$ and $\mathrm{SO}$ emission (left and right panels, respectively). $\mathrm{N}_{2} \mathrm{D}^{+}$emission has been integrated over velocities -2.56 to $5.49 \mathrm{~km} \mathrm{~s}^{-1}$, while the $\mathrm{SO}$ emission has been integrated over velocities 0.90 to $4.21 \mathrm{~km} \mathrm{~s}^{-1}$. Contour levels for the $\mathrm{CH}_{3} \mathrm{OH}$ and the continuum emission are $-5,5,10,20,35$, and 50 times the rms $\left(6.8 \times 10^{-2} \mathrm{Jy} /\right.$ beam km s${ }^{-1}$ and $1.11 \times 10^{-3} \mathrm{Jy} /$ beam, respectively $)$. For SO, contour levels are -3 , $3,6,10,15$, and 20 times the rms $\left(6.7 \times 10^{-2} \mathrm{Jy} /\right.$ beam $\left.\mathrm{km} \mathrm{s}^{-1}\right)$. The synthesized beam for the $\mathrm{CH}_{3} \mathrm{OH}$ and SO images is 4 !' $88 \times 33^{\prime \prime} 19$ at $81^{\circ}$ for $\mathrm{ND}_{3}(\mathrm{lower}$ right corner of the right panel) and 5". $04 \times 33^{\prime \prime} 16$ at $80^{\circ}$ for $970 \mu \mathrm{m}$ continuum (shown in the lower right corner). Symbols are the same as in Figure 6 .

emission to the southwest. VLBI water maser observations agree with this picture, showing a very well-defined bow-shock among the redshifted masers to the southwest (A. Wootten 2016, private communication). Source A-E, in orbit around A-W, also shows maser outflow, approximately parallel to that in $\mathrm{A}-\mathrm{W}$ and in the same sense, i.e., redshifted emission to the southwest. On larger scales, the overall complexity of the L1689N region makes the interpretation of the outflow difficult. However, one clear conclusion is that there is a lot of moderate-velocity blueshifted gas in the immediate vicinity of the prestellar core, and the change in the observed $\mathrm{NH}_{2} \mathrm{D}$ line velocity and width is direct evidence that this gas is interacting with the prestellar core.

Interestingly, the polarity of both compact $\mathrm{CO}$ outflows seen in the SMA data (Girart et al. 2014) seems opposite to that of the large-scale outflows seen in the earlier lower-resolution single-dish data (Mizuno et al. 1990; Lis et al. 2002a; Stark et al. 2004; compare, e.g., Figure 1 of Girart et al. 2014 and Figure 17 of Stark et al. 2004). The northern peak of the blueshifted water emission appears to be a part of the main east-west outflow detected in the SMA CO data. VLBA observations of the water maser proper motions (Wootten et al. 1999) also show that the IRAS 16293A outflow proceeds red NE, blue SW, from within a few astronomical units of the sources.

The key observational aspects of the new data presented here that have to be satisfactorily explained by a source model are the following: (a) the change in the $\mathrm{NH}_{2} \mathrm{D}$ line center velocity and width across the core (Figure 5); (b) the apparent offset between the peak of the $970 \mu \mathrm{m}$ dust continuum emission and the emission of deuterated molecular tracers in the ACA data (Figure 6).
One possible explanation is that the prestellar core in L1689N is simply a pre-existing density enhancement that had formed independently of IRAS 16293. This region is characterized by some of the highest deuteration levels known in galactic sources. For example, it is one of a handful of sources where triply deuterated ammonia has been detected (Roueff et al. 2005). Such cores are generally rare, but Barnard 1 offers another example of a similar region. Here, a dense prestellar core, B1b (also referred to as SMM1), with very high deuteration levels, which has been suggested to host the first hydrostatic core (Gerin et al. 2015), is also located in close proximity to more evolved protostellar sources, B1a (SMM6) and B1c (SMM2), driving pronounced outflows (Hatchell et al. 2007; Hiramatsu et al. 2010). High deuteration levels require a low temperature, high density, and consequently high $\mathrm{CO}$ depletion. Such conditions are found in isolated prestellar cores, e.g., L1544, which are characterized by a centrally peaked density distribution that can be traced well by dust continuum emission. However, our ACA data reveal no bright compact dust continuum sources in the northern part of the core, where the strongest emission of deuterated molecular tracers is seen in single-dish maps.

Lis et al. (2002a) suggested that this region may be a part of the ambient cloud that is pushed and compressed by the outflow. The shock-compressed, dense gas then cools efficiently to low temperatures, and the gas-phase chemistry quickly drives up abundances of deuterated molecular species. This scenario is in fact consistent with the observations presented here. The blue lobe of the IRAS 16293 east-west outflow impacts the prestellar core from the back. The shock could have already propagated through the northeastern part of the core, where we now see narrow, undisturbed line profiles in the cold, compressed post-shock gas, blueshifted 

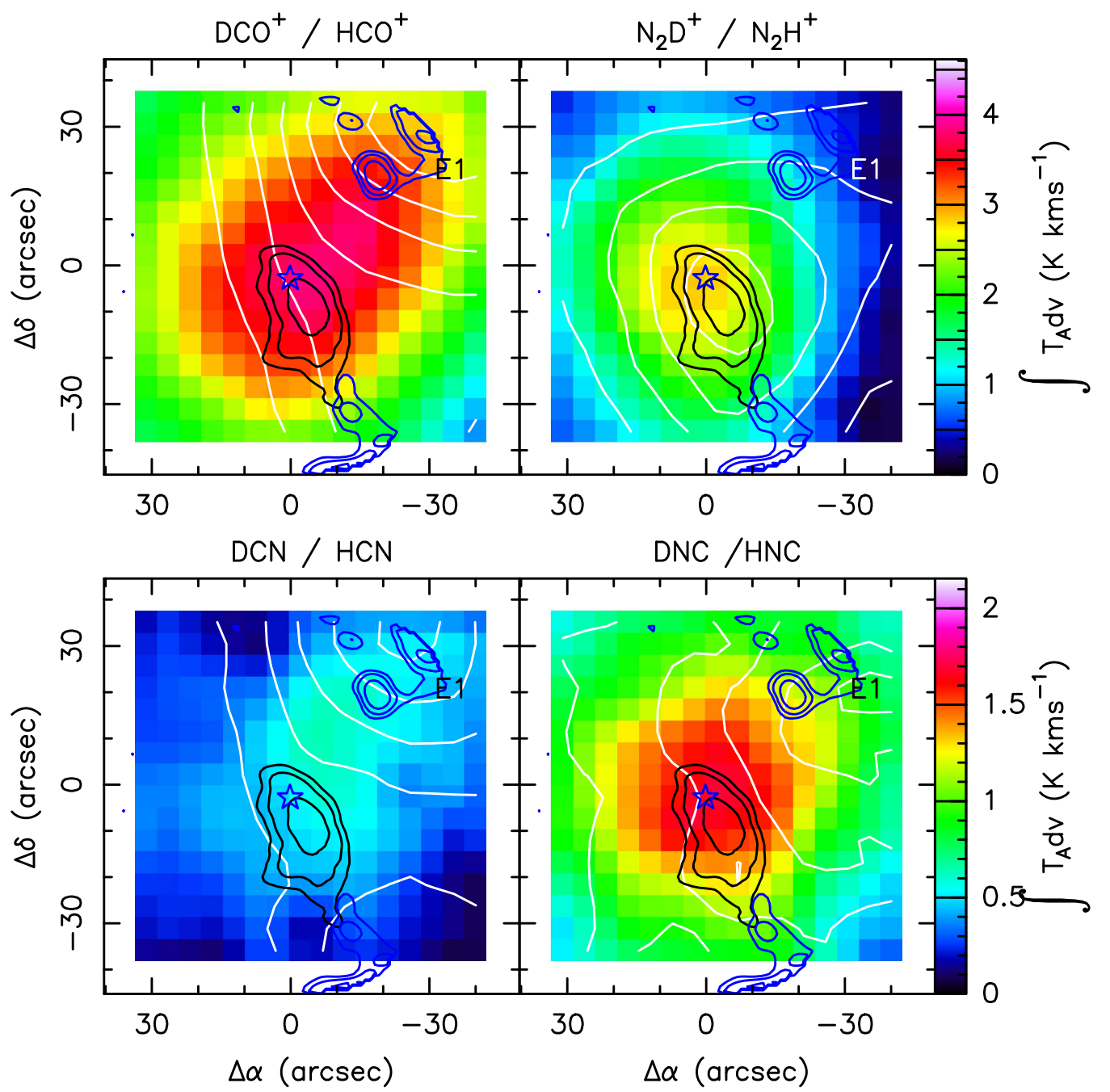

Figure 9. CSO maps of deuterated species in L1689N (color images) with overlaid white contours of their hydrogenated counterpars. $\mathrm{N}_{2} \mathrm{D}^{+}$and $\mathrm{DNC}$ are the best tracers of the prestellar core with $\mathrm{N}_{2} \mathrm{D}^{+}$being more centrally peaked. Both $\mathrm{DCO}^{+}$and $\mathrm{DCN}$ emission show extensions toward the E1 source in the northwest. Black contours show the distribution of $\mathrm{NH}_{2} \mathrm{D}$ as observed with the ACA, while the blue contours show the distribution of $\mathrm{CH}_{3} \mathrm{OH}$. Contour levels are 5-17.5 with a step of $2.5 \mathrm{~K} \mathrm{~km} \mathrm{~s}^{-1}$ for $\mathrm{HCO}^{+}, 2.5-7$ with a step of $1.5 \mathrm{~K} \mathrm{~km} \mathrm{~s}^{-1}$ for $\mathrm{N}_{2} \mathrm{H}^{+}, 2-8$ with a step of $1.5 \mathrm{~K} \mathrm{~km} \mathrm{~s}^{-1}$ for $\mathrm{HCN}$, and $1-3$ with a step of $0.5 \mathrm{~K} \mathrm{~km} \mathrm{~s}^{-1}$ for $\mathrm{HCO}^{+}$. ACA contour levels are 10,20, and 40 times the rms. The star marks the location of the $\mathrm{NH}_{2} \mathrm{D}$ emission peak observed with HIFI.

with respect to the systemic velocity of the ambient cloud. An order of magnitude density enhancement, as compared to the ambient cloud, expected in the post-shock gas produces conditions that are propitious for the high deuteration levels observed. The west/southwest part of the core is, however, still interacting with the outflow, hence the disturbed appearance of the molecular line profiles with broader line widths. The shock to the north/northwest of the core (traced by, e.g., the $\mathrm{SiO}$ or $\mathrm{CH}_{3} \mathrm{OH}$ emission) may be associated with a higher-velocity component of the flow, possibly a jet or jet remnant, as the highest blueshifted water and $\mathrm{CO}$ emission occurs there. There may also be a pre-existing density enhancement at this location which happens to lie in the path of the higher-velocity component of the flow, which was further compressed and heated up by the shock. This would explain the strong emission of high-density molecular tracers such as $\mathrm{HCO}^{+}, \mathrm{HCN}, \mathrm{HNC}$, and $\mathrm{SiO}$ in the single-dish images (Figure 9; Hirano et al. 2001). This region also stands out in the ACA $\mathrm{CH}_{3} \mathrm{OH}$ image and thus shows the classic outflow-dense gas interaction signatures.
The apparent offset between the dust continuum peak, as observed with the ACA, and the emission of deuterated molecular tracers is another intriguing aspect. Such offsets have been observed in other prestellar cores (e.g., L183; Tiné et al. 2000), or in outflows (e.g., L1157; Fontani et al. 2014). The dust continuum emission is typically taken as a measure of the $\mathrm{H}_{2}$ column density, indicating the location of the densest central parts of prestellar cores. However, possible variations in the dust temperature may strongly affect this interpretation. In fact, multi-wavelength Spitzer and Herschel continuum data (Pagani et al. 2016) indicate a NW-SE temperature gradient across the prestellar core, with colder dust located in the north. While the average dust temperature in a single-dish beam is $\sim 16 \mathrm{~K}$ (Stark et al. 2004), this may be dominated by the outer surface layers and the dust temperature in the central, most shielded regions may be significantly lower (see Bacmann et al. 2016). The ACA $850 / 970 \mu \mathrm{m}$ flux ratio map shows no evidence for a temperature gradient across the compact source. However, the $\mathrm{S} / \mathrm{N}$ in our $850 \mu \mathrm{m}$ continuum image is too low to draw definitive conclusions. 

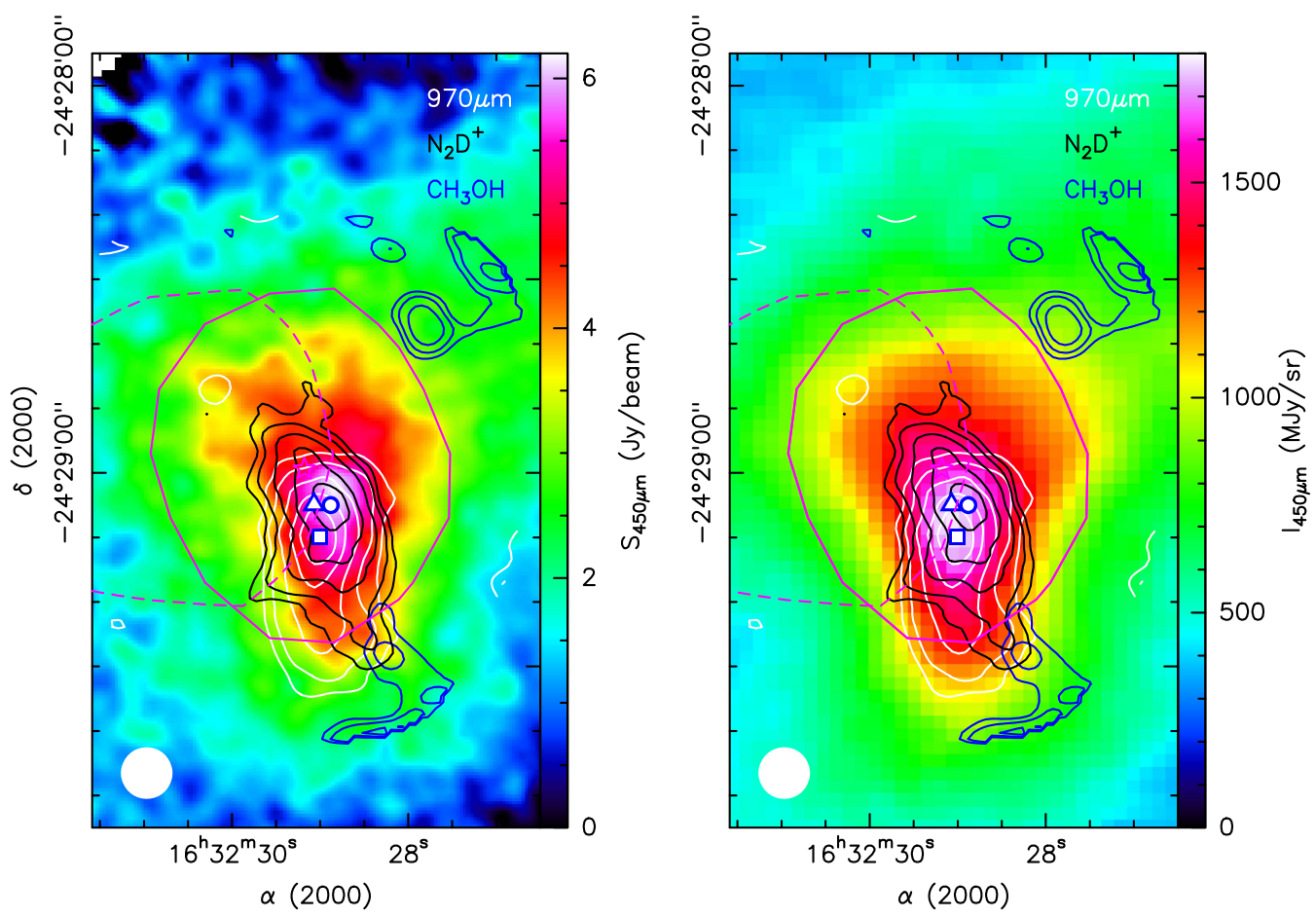

Figure 10. Color images of the single-dish $450 \mu \mathrm{m}$ dust continuum emission toward the prestellar core, observed with SCUBA (Stark et al. 2004; left panel) and SCUBA-2 (Pattle et al. 2015; right panel), with overlaid contours of the $970 \mu \mathrm{m}$ continuum, $\mathrm{N}_{2} \mathrm{D}^{+}$, and $\mathrm{CH}_{3} \mathrm{OH}$ emission observed with the ACA (white, black, and blue contours, respectively). Contour levels are the same as in Figures 6 and 8. The solid magenta contour shows the location of the $\mathrm{NH}_{2} \mathrm{D}$ emission, as observed by HIFI, while the dashed magenta contour outlines the location of the $\mathrm{H}_{2} \mathrm{D}^{+}$emission (Pagani et al. 2016; 60\% contour levels).

Another important aspect is that some of the extended continuum emission may simply be filtered out in the ACA data. Figure 10 shows $450 \mu \mathrm{m}$ SCUBA and SCUBA-2 dust continuum emission images toward the prestellar core (Stark et al. 2004; Pattle et al. 2015, left and right panels, respectively) with overlaid contours of the $970 \mu \mathrm{m}$ continuum and $\mathrm{N}_{2} \mathrm{D}^{+}$emission observed with the ACA (green and black contours, respectively). The single-dish dust continuum emission in the SCUBA image correlates well with the $\mathrm{N}_{2} \mathrm{D}^{+}$emission and is clearly shifted to the north with respect to the $970 \mu \mathrm{m}$ ACA continuum peak. The lower angular resolution $870 \mu \mathrm{m}$ SCUBA image shows a similar offset. However, the $450 \mu \mathrm{m}$ SCUBA-2 image peaks closer to the ACA continuum peak. The small differences between the two images may be caused by limited $\mathrm{S} / \mathrm{N}$ or pointing offsets. The integrated $345 \mathrm{GHz}$ ACA flux is only $0.72 \mathrm{Jy}$, as compared to the peak $870 \mu \mathrm{m}$ broadband flux of $1.4 \mathrm{Jy}$ in the $14^{\prime \prime}$ JCMT beam (Stark et al. 2004). The minimum baseline of the ACA is $8.7 \mathrm{~m}$ for a transiting source. The effective baseline depends on the hour angle, but for a transiting source the ALMA Technical Handbook provides a value of 13."8 for the largest angular scale recovered by the interferometer. This strongly suggests that the ACA continuum image may not represent the true $\mathrm{H}_{2}$ column density distribution in the prestellar core. It appears instead that the northern, quiescent part of the core is characterized by spatially extended continuum emission, which is mostly resolved out by the interferometer, while the southern part is more compact and centrally peaked. This is consistent with the interpretation outlined above in which the northern part of the core is compressed, high-density, cold post-shock gas, which is relatively uniformly distributed and not yet fragmenting to form new stars. This shows that good care should be taken when interpreting ALMA observations of extended sources, even with the ACA, when the total-power data are not included.

Interestingly, the $\mathrm{H}_{2} \mathrm{D}^{+}$emission shows a very distinct morphology compared to any of the tracers studied here, peaking largely to the east/northeast (see Figure 1 of Pagani et al. 2016 and the dashed magenta contour in Figure 10). Pagani et al. (2016) argue that this is in fact the location of the coldest, highest column density gas in the region. We note that a weak $970 \mu \mathrm{m}$ continuum peak, with a flux density of $6.9 \mathrm{mJy} /$ beam (a $6 \sigma$ detection) is present within the $\mathrm{H}_{2} \mathrm{D}^{+}$ emission region, which can be identified with source 86 (SMA 22) of Pattle et al. (2015). An extension in this direction is also seen in the $870 \mu \mathrm{m}$ SCUBA image. More sensitive observations with the main ALMA array are required to determine the exact nature of this source.

Another important factor is that broadband, single-dish continuum images may be contaminated by molecular line emission. As shown in Figure 9, only the classical tracers of dense cold gas, namely, deuterated species and $\mathrm{N}_{2} \mathrm{H}^{+}$, peak toward the prestellar core. Other molecular tracers largely avoid the core and peak instead toward the E1 shock. However, a weak $\mathrm{H}^{13} \mathrm{CO}^{+} 4-3$ emission peak associated with the northern part of the core was detected by Gerin et al. (2006). The blueshifted $\mathrm{CO}$ emission to the north of the prestellar core may also influence the broadband continuum images, given the relatively broad line widths. Molecular line contamination may thus effectively shift the broadband continuum peak to the north, away from the compact continuum source seen in the ACA image, explaining the observed morphology. However, one clear conclusion is that there are no strong compact continuum sources embedded in the northern, quiescent part of the prestellar core. 
We note that $\mathrm{ND}_{3}$ emission was detected with a high $\mathrm{S} / \mathrm{N}$ toward the prestellar core in the single-dish CSO data (Roueff et al. 2005) with an integrated line intensity in the $25^{\prime \prime}$ CSO beam of $0.29 \mathrm{~K} \mathrm{~km} \mathrm{~s}^{-1}$ (main-beam brightness temperature), or $\sim 9.4 \mathrm{Jy} \mathrm{km} \mathrm{s}^{-1}$. The integrated $\mathrm{ND}_{3}$ flux in the ACA image is $6.8 \mathrm{Jy} \mathrm{km} \mathrm{s}^{-1}$, indicating that a significant fraction of the single-dish flux is in fact recovered by the interferometer.

\section{SUMMARY}

The combined single-dish and interferometric observations of L1689N presented here show clear evidence of an interaction between the prestellar core seen in the dust continuum and line emission of deuterated molecular tracers, notably $\mathrm{NH}_{2} \mathrm{D}, \mathrm{ND}_{3}, \mathrm{~N}_{2} \mathrm{D}^{+}$, and $\mathrm{DNC}$, and the molecular outflow emanating from the nearby solar-type protostar IRAS 16293. Herschel observations of water and singly deuterated ammonia show that the outflow wraps around and largely avoids the prestellar core. A shift in the $\mathrm{NH}_{2} \mathrm{D}$ line velocity and width is seen across the core, with the narrowest line profiles observed toward the north-eastern part of the core. We suggest that the shock associated with the outflow has already propagated through this part of the core and we see quiescent, cold, shock-compressed, dense gas that is blueshifted with respect to the systemic velocity of the cloud. The western/ south-western part of the core is still interacting with the outflow, as evidenced by the broader, more disturbed line profiles.

The $\mathrm{N}_{2} \mathrm{D}^{+}$emission observed with the ACA shows an elongated distribution, extending south/southwest from the single-dish $\mathrm{NH}_{2} \mathrm{D}$ peak. $\mathrm{ND}_{3}$ shows a similar morphology, although the peak of the emission is shifted $\sim 2$ ". 5 east compared to $\mathrm{N}_{2} \mathrm{D}^{+}$, possibly due to optical depth effects. The $970 \mu \mathrm{m}$ dust continuum emission observed with the ACA is offset $\sim 5^{\prime \prime}$ (one ACA synthesized beam) to the south with respect to the $\mathrm{N}_{2} \mathrm{D}^{+}$and $\mathrm{ND}_{3}$ emission peaks. A possible explanation is that the material in the northern part of the core is largely uniformly distributed and has not yet fragmented into compact sources that can lead to a second generation of star formation in the region. The continuum emission is thus largely resolved out by the interferometer. However, a compact dust source with a size of $\sim 1100$ au and an $\mathrm{H}_{2}$ mass of $\sim 0.2-0.4 M_{\odot}$ is present in the southern part of the core. The sensitivity of the current $\mathrm{ACA} \mathrm{ND}_{3}$ data is not sufficient to determine whether this source is collapsing and the high $\mathrm{S} / \mathrm{N} \mathrm{N}_{2} \mathrm{D}^{+}$spectra are affected by the complex HFS.

The observations presented here provide clear evidence of a physical interaction between the prestellar core in L1689N and the blue lobe of one of the outflows driven by IRAS16293. How frequent such interactions may be in nearby low-mass star-forming regions is difficult to answer quantitatively. Hiramatsu et al. (2010) suggested that a similar interaction may occur in Barnard 1. They concluded that outflows cannot sustain the turbulence in the main Barnard 1 core, but they are energetic enough to compensate the turbulent energy dissipation in the neighboring, more evolved star-forming region NGC1333. Recent observations of Serpens South and NGC1333 (Plunkett 2015) also suggest that outflows are energetically important and provide sufficient energy to sustain turbulence early in the protocluster formation process without disrupting the entire cluster. Observations of low-mass starforming regions often show bright $\mathrm{DCO}^{+}$emission, which is assumed to trace cold, dense gas, coinciding with lobes of high- velocity $\mathrm{CO}$ emission rather than dust continuum peaks, marking the locations of embedded protostars (e.g., Lis et al. 2004). Most of these $\mathrm{DCO}^{+}$sources have not been studied in enough detail to determine whether they are true interaction regions, or whether they in fact harbor prestellar cores, as is the case in L1689N. Roueff et al. (2005) also pointed out that $\mathrm{NH}_{2} \mathrm{D}$ and $\mathrm{ND}_{3}$ emission often does not peak at the location of embedded protostars, but instead at offset positions where outflow interactions may occur. In their sample of six sources where $\mathrm{ND}_{3}$ emission has been detected, L1689N is the only region where a physical interaction has been clearly demonstrated, but an outflow interaction has also been suggested in Barnard 1 (Hiramatsu et al. 2010). Another candidate source is located in NGC1333, at the edge of the blue lobe of the powerful molecular outflow associated with the $\mathrm{HH} 7-11$ jet (Lis et al. 2004). However, this source has not yet been studied sufficiently to make a convincing case.

In conclusion, the observations presented here demonstrate the utility of the fundamental rotational transitions of deuterated ammonia as an additional tracer of the deeply embedded, prestellar phase of star formation. Collisional crosssections of $\mathrm{NH}_{2} \mathrm{D}, \mathrm{ND}_{2} \mathrm{H}$, and $\mathrm{ND}_{3}$ with $\mathrm{H}_{2}$ are now available (Daniel et al. 2014, 2016b), allowing detailed modeling using state-of-the art radiative transfer codes. The lines are accessible to current ground-based submillimeter facilities, in particular ALMA, offering new insights into the early phases of the star formation process.

HIFI has been designed and built by a consortium of institutes and university departments from across Europe, Canada and the United States (NASA) under the leadership of SRON, Netherlands Institute for Space Research, Groningen, The Netherlands, and with major contributions from Germany, France and the US. Support for this work was provided by NASA (Herschel OT funding) through an award issued by JPL/Caltech. This paper makes use of the following ALMA data: ADS/JAO.ALMA\#2012.1.00178.S. ALMA is a partnership of ESO (representing its member states), NSF (USA), and NINS (Japan), together with NRC (Canada), NSC, and ASIAA (Taiwan), and KASI (Republic of Korea), in cooperation with the Republic of Chile. The Joint ALMA Observatory is operated by ESO, AUI/NRAO, and NAOJ. This work is based upon observations with the the Caltech Submillimeter Observatory, operated by the California Institute of Technology. The National Radio Astronomy Observatory is a facility of the National Science Foundation operated under cooperative agreement by Associated Universities, Inc. C.M.W. would like to acknowledge financial support from the Science Foundation Ireland (Grant 13/ERC/I12907). We thank the anonymous referee for helpful comments.

\section{REFERENCES}

André, P., Di Francesco, J., Ward-Thompson, D., et al. 2014, in Protostars and Planets VI, ed. H. Beuther et al. (Tucson: Univ. Arizona Press), 27 André, P., Men'shchikov, A., Bontemps, S., et al. 2010, A\&A, 518, L102 Bacmann, A., Daniel, F., Caselli, P., et al. 2016, A\&A, 587, 26 Ballesteros-Paredes, J., \& Hartmann, L. 2007, RMxAA, 43, 123 Belloche, A., André, P., Despois, D., \& Blinder, S. 2002, A\&A, 393, 927 Bergin, E. A., \& Tafalla, M. 2007, ARA\&A, 45, 339 Caselli, P., Keto, E., Bergin, E. A., et al. 2012, ApJL, 759, L37 Caselli, P., Vastel, C., Ceccarelli, C., et al. 2008, A\&A, 492, 703 Castets, A., Ceccarelli, C., Loinard, L., et al. 2001, A\&A, 375, 40 Ceccarelli, C., Caselli, P., Bockelée-Morvan, D., et al. 2014, in Protostars and Planets VI, ed. H. Beuther et al. (Tucson: Univ. Arizona Press), 859 
Crapsi, A., Caselli, P., Walmsley, M. C., \& Tafalla, M. 2007, A\&A, 470, 221

Daniel, F., Faure, A., Wiesenfeld, L., et al. 2014, MNRAS, 444, 2544

Daniel, F., Faure, A., Pagani, L., et al. 2016a, A\&A, 592, A45

Daniel, F., Rist, C., Faure, A., et al. 2016b, MNRAS, 457, 1535

De Graauw, Th., Helmich, F. P., Phillips, T. G., et al. 2010, A\&A, 518, L6

di Francesco, J., Evans, N. J., II, Caselli, P., et al. 2007, in Protostars and

Planets V, ed. V. B. Reipurth, D. Jewitt, \& K. Keil (Tucson: Univ. Arizona Press), 17

Dunham, M. M., Stutz, A. M., Allen, L. E., et al. 2014, in Protostars and Planets VI, ed. H. Beuther et al. (Tucson: Univ. Arizona Press), 195

Fedoseev, G., Ioppolo, S., \& Linnartz, H. 2015, MNRAS, 446, 449

Flower, D. R., Pineau des Forêts, G., \& Walmsley, C. M. 2006, A\&A, 456, 215

Fontani, F., Codella, C., Ceccarelli, C., et al. 2014, ApJL, 788, L43

Gerin, M., Lis, D. C., Philipp, S., et al. 2006, A\&A, 454, L63

Gerin, M., Pety, J., Fuente, A., et al. 2015, A\&A, 577, L2

Girart, J. M., Esalella, R., Palau, A., et al. 2014, ApJL, 780, L11

Hatchell, J., Fuller, G. A., \& Richer, J. S. 2007, A\&A, 472, 127

Hennebelle, P., \& Chabrier, G. 2008, ApJ, 684, 395

Hennebelle, P., \& Chabrier, G. 2009, ApJ, 702, 1428

Hennebelle, P., \& Fromang, S. 2007, A\&A, 477, 9

Hennebelle, P., \& Teyssier, R. 2007, A\&A, 477, 25

Hiramatsu, M., Hirano, N., \& Takakuwa, S. 2010, ApJ, 712, 778

Hirano, N., Mikami, H., Umemoto, T., Yamamoto, S., \& Taniguchi, Y. 2001, ApJ, 547, 899

Könyves, V., André, P., Men'shchikov, A., et al. 2010, A\&A, 518, L106

Lefèvre, C., Pagani, L., Juvela, M., et al. 2014, A\&A, 572, A20

Lefèvre, C., Pagani, L., Min, M., Poteet, C., \& Whittet, D. 2016, A\&A, 585, L4

Lis, D. C., Gerin, M., Phillips, T. G., \& Motte, F. 2002a, ApJ, 569, 322

Lis, D. C., Gerin, M., Roueff, E., Vastel, C., \& Phillips, T. G. 2006, ApJ, 636, 916

Lis, D. C., Gerin, M., Roueff, E., Phillips, T. G., \& Poelman, D. R. 2008, Ap\&SS, 313, 77

Lis, D. C., Gerin, M., Roueff, E., \& Phillips, T. G. 2004, in The Dense Interstellar Medium in Galaxies, ed. S. Pfalzner et al. (Berlin: Springer), 487

Lis, D. C., Roueff, E., Gerin, M., et al. 2002b, ApJL, 571, L55

Loinard, L., Rodríguez, L. F., D’Alessio, P., Rodríguez, M. I., \& González, R. F. 2007, ApJ, 670, 1353

Loinard, L., Torres, R. M., Mioduszewski, A. J., \& Rodríguez, L. F. 2008, ApJL, 675, L29

Maury, A. J., André, P., Hennebelle, P., et al. 2010, A\&A, 512, A40

Menten, K. M., Serabyn, E., Güsten, R., \& Wilson, T. L. 1987, A\&A, 177, L57

Mizuno, A., Fukui, Y., Iwata, T., et al. 1990, ApJ, 356, 184

Molinari, S., Swinyard, B., Bally, J., et al. 2010, A\&A, 518, L100

Motte, F., André, P., \& Neri, R. 1998, A\&A, 336, 150
Mouschovias, T. Ch., Morton, S. A., et al. 1991, ApJ, 371, 296

Ott, S. 2010, in ASP Conf. Ser. 434, Astronomical Data Analysis Software and Systems XIX, ed. Y. Mizuno, K. I. Morita, \& M. Ohishi (San Francisco, CA: ASP), 139

Pagani, L., Bacmann, A., Cabrit, S., \& Vastel, C. 2007, A\&A, 467, 179

Pagani, L., Lefèvre, C., Juvela, M., Pelkonen, V.-M., \& Schuller, F. 2015, A\&A, 574, L5

Pagani, L., Lefèvre, C., Parise, B., et al. 2016, in Conditions and Impact of Star Formation: From Lab to Space, ed. R. Simon et al. (Paris: EDP Sciences)

Pagani, L., Salez, M., \& Wannier, P. G. 1992, A\&A, 258, 479

Pagani, L., Steinacker, J., Bacmann, A., Stutz, A., \& Henning, T. 2010, Sci, 329, 1622

Pagani, L., Vastel, C., Hugo, E., et al. 2009, A\&A, 494, 623

Parise, B., Leurini, S., Schilke, P., Roueff, E., Thorwirth, S., \& Lis, D. C. 2009, A\&A, 508, 737

Pattle, K., Ward-Thompson, D., Kirk, J. M., et al. 2015, MNRAS, 450, 1094

Pilbratt, G. L., Riedinger, J. R., Passvogel, T., et al. 2010, A\&A, 518, L1

Plunkett, A. L. D. 2015, PhD thesis, Yale Univ.

Roberts, H., \& Millar, T. J. 2000, A\&A, 364, 780

Roelfsema, P. R., Helmich, F. P., Teyssier, D., et al. 2012, A\&A, 537, A17

Roueff, E., Lis, D. C., van der Tak, F. F. S., Gerin, M., \& Goldsmith, P. F. 2005, A\&A, 438, 585

Roueff, E., Parise, B., \& Herbst, E. 2007, A\&A, 464, 245

Shu, F., Adams, F. C., \& Lizano, S. 1987, ARA\&A, 25, 23

Stark, R., Sandell, G., Beck, S. C., et al. 2004, ApJ, 608, 341

Steinacker, J., Pagani, L., Bacmann, A., \& Guieu, S. 2010, A\&A, 511, A9

Tafalla, M., Myers, P. C., Caselli, P., Walmsley, C. M., \& Comito, C. 2002, ApJ, 569, 815

Tiné, S., Roueff, E., Falgarone, E., Gerin, M., \& Pineau des Forêts, G. 2000, A\&A, 356, 1039

van der Tak, F. F. S., Caselli, P., \& Ceccarelli, C. 2005, A\&A, 439, 195

Vastel, C., Caselli, P., Ceccarelli, C., et al. 2006, ApJ, 645, 1198

Vastel, C., Caselli, P., Ceccarelli, C., et al. 2012, A\&A, 547, A33

Ward-Thompson, D., André, P., Crutcher, R., et al. 2007, in Protostars and Planets V, ed. B. Reipurth, D. Jewitt, \& K. Keil (Tucson: Univ. Arizona Press), 33

Wootten, A., Claussen, M., Marvel, K., \& Wilking, B. 1999, in The Physics and Chemistry of the Interstellar Medium, ed. V. Ossenkopf, J. Stutzki, \& G. Winnewisser (Herdecke: GCA-Verlag), 295

Wootten, A., \& Loren, R. B. 1987, ApJ, 317, 220

Yeh, S. C. C., Hirano, N., Bourke, T. L., et al. 2008, ApJ, 675, 454 